\title{
Nonlinear Analysis on Seismic Site Response of Fuzhou Basin, China
}

\author{
by Guoxing Chen, Dandan Jin, Jiao Zhu, Jian Shi, and Xiaojun Li
}

\begin{abstract}
Based on the parallel computing cluster platform of the ABAQUS software, a large-scale 2D finite-element refined nonlinear modeling approach was used to study seismic site effects in the Fuzhou basin, such as peak ground acceleration (PGA), spectral acceleration, duration, and acceleration transfer functions. A 1D equivalent linear wave propagation analysis was conducted also, with Proshake for supplementation and calibration. The simulation results demonstrated the following. (1) PGA amplification factors exhibited spatial variation characteristics that varied both laterally and with depth and exhibited a nonmonotonic decreasing characteristic with soil depth. From the 2D results, a greater motion amplification and focusing effect for some shallow soil layers was observed where there was significant fluctuation of the underlying bedrock interface. The moderate- and long-period ground-motion components were obviously amplified, but were was less pronounced in the 1D result. (2) For low-, moderate-, and high-level earthquakes, the surface PGA amplification factors were $1.4-2.5,1.3-2.0$, and 1.1-2.0, respectively, and the predominant periods of the basin were $0.35-0.65 \mathrm{~s}, 0.40-0.75 \mathrm{~s}$, and $0.50-1.05 \mathrm{~s}$, respectively. (3) Ground-motion durations were prolonged to different degrees, which were closely related to the characteristics of seismic bedrock motions. (4) The sensitive frequency band of the seismic site response was from 0.5 to $2 \mathrm{~Hz}$. (5) Ground-motion amplification in the downtown section of the city of Fuzhou was generally larger. To a certain extent, the 2D results reflect the influences of the surface topography relief, the fluctuation of the underlying bedrock interface, and lateral heterogeneity of soils on seismic-wave propagation.
\end{abstract}

\section{Introduction}

Local site conditions have a profound influence on seismic damage; this is well-known among seismologists and civil engineers as the site effect. Many earthquake damage investigations and the results of theoretical research indicated the local variations of topography and sediment deposit may affect the propagation of seismic waves, resulting in a significant difference in the spatial variation of ground motions (Di Fiore, 2010; Lanzo et al., 2011; Assimaki et al., 2012; Héloïse, 2012; Assimaki and Jeong, 2013; Sheng et al., 2013). Damage to property and loss of life from destructive earthquakes frequently result from local site conditions that affect the propagation of seismic waves; consequently, any attempt to undertake seismic design for a project must take the local site conditions into account. Based on the seismic damage data for the 1906 San Francisco, California, earthquake, Wood argued that the local site conditions emerge as a dominant factor controlling the amplification of ground motions and the concentration of damage during earthquakes (Wood, 1916). This was also verified by seismic damage investigation records of such earthquake events as the $1923 M_{\mathrm{s}} 8.2$ Kanto earthquake in Japan, the $1966 M_{\mathrm{s}} 7.2$ Xingtai earthquake in China, the $1976 M_{\mathrm{s}} 7.8$ Tangshan earthquake in China, the $1985 M_{\mathrm{s}} 8.1$ Michoacan earthquake in Mexico, the $1999 M_{\mathrm{s}} 7.8$ Kocaeli earthquake in Turkey, and the $1999 M_{\mathrm{s}}$ 7.6 Chi-Chi earthquake in Taiwan, China. The influence of local site conditions on the site effect has been studied since the 1960s in China, especially after the $1970 M_{\mathrm{s}} 7.7$ Tonghai earthquake; its influence was further emphasized in the investigation of strong motion (Qian et al., 1984).

As we know, many large cities are located in sedimentary basins, where the geological conditions of the local sites are spatially complex; the spatial variations of ground motion and the seismic damage pattern in a sedimentary basin aroused great interest for the seismologists and civil engineers. For example, during the $1985 M_{\mathrm{s}} 8.1$ Michoacan earthquake in Mexico, the most serious damage was in the Mexico City area, which was approximately $400 \mathrm{~km}$ from the epicenter (Somerville and Graves, 1993). The disaster left 5500 people dead, 3500 people unaccounted for, 30,000 people injured, and 1132 houses destroyed. Seismic damage was serious in the central district and almost nonexistent in the outskirts. By analyzing the ground motion and microtremor records in Mexico City (Singh et al., 1988; Campillo et al., 1989), seismologists proposed that the predominant periods of soft soil sites in the central area of the city was $2 \mathrm{~s}$, and the heavy damage in Mexico City was primarily caused by the 
horizontal magnification of the ground motion with a predominant period of 2-3 s. This finding shows that the horizontal seismic waves are reflected and refracted repeatedly during the wave propagation through the deep soft soil in the basin, which significantly extends the amplitude and prolongs the duration of ground motions. The fundamental period of the buildings is close to the predominant period of the site, thus the induced resonance-like phenomenon led to serious building damage.

This peculiar phenomenon of severe earthquake damage in Mexico City plays an important role in the study of basin effect. Bard et al. (1988), Semblat et al. (2000), and Stewart et al. (2001) indicated that there are always structural soft soils with peculiar dynamic nonlinear characteristics, which may remarkably amplify long-period ground motions and prolong the duration of strong ground motions in soft soil layers. Chávez-García et al. (2000) and Semblat et al. (2002) noted that the underlying bedrock geometry in a bowl-shaped undulating basin focused seismic waves on specific locations within the basin. The ground motions are amplified by constructive interference between the surface or diffracted waves generated at the basin edges and the direct waves. Moczo (1989), Moczo and Bard (1993), and Moczo et al. (1996) used a finite-difference technique for $S H$ waves in 2D media using irregular grids, as applied to the seismic response problem. Makraa et al. (2005) presented the main features of the seismic response for a sedimentary valley; for example, they showed that locally generated surface waves made significant contributions to ground motions, and the lateral heterogeneity at the valley had a remarkable influence on ground motions. Sun et al. (2008) noted that basin geometry and special site conditions have important influences on ground-motion amplification. Sedimentary soil significantly amplified the incident shear waves, and the amplification factors of peak ground acceleration (PGA) were 2.5-5. On the whole, the interior parts of the basins adjacent to the edges showed larger PGAs than in the central parts, and the duration of the ground motions at the basin edges was prolonged as a result of the generation of surface waves at the basin edges. Gélis and Bonilla $(2012,2014)$ assessed the propagation of seismic waves in a basin, considering linear and nonlinear constitutive models. The basin response depended on the soil properties, the site profile geometry, the impedance contrast, the constitutive model of soil, and the characteristics of the input motions. These researchers showed that high shear-strain values were located in a layered basin at the bottom of superficial layers where high impedance contrasts led to wave amplification. Nonlinearity generally enhances wave amplification at the bottoms of the layers.

At present, the refined nonlinear numerical simulation for 2D seismic response analysis of large-scale basins is still limited. In a flat site with a horizontal layer of soil, local amplification can be reasonably inferred using simple 1D wave propagation models. However, if the surface topography relief and underlying bedrock interface undulate intensely, the lateral heterogeneity of soils in the basin may give rise to focusing effects on soil amplification and to local generation of surface waves, so the estimates for local amplification using a 1D wave propagation model may be inaccurate. As a result, 2D methods for investigating seismic site effects of complex basins have gained popularity among researchers.

In this article, based on the explicit finite-element method, a large-scale 2D refined nonlinear modeling approach for the Fuzhou basin with the fluctuation of underlying bedrock interface and lateral heterogeneity soil was used in the time domain to study the characteristics of ground motions for different seismic levels, such as PGA, spectral acceleration, duration, the acceleration transfer function, and the predominant period. In addition, 1D equivalent-linear wave propagation analyses were conducted for supplementation and calibration. Finally, conclusions regarding the primary features of seismic site effects in the Fuzhou basin were discussed.

\section{Engineering Geological Conditions and the Earthquake Environment of Fuzhou Basin}

The Fuzhou basin is located in the east-coastal areas of Fujian Province, China, lying at longitude $118^{\circ} 08^{\prime}-120^{\circ} 31^{\prime} \mathrm{E}$ and latitude $25^{\circ} 15^{\prime}-26^{\circ} 29^{\prime} \mathrm{N}$, on the lower reaches of the Minjiang River and is a Cenozoic basin approximately $30 \mathrm{~km}$ from the sea. There are always alluvial-deluvial and alluvialmarine soils after the Late Pleistocene in Cenozoic fault basins and gulfs. The Fuzhou basin is mainly an alluvial plain, with undulating low mountains and hills interspersed (see Fig. 1). Based on regional geological survey borehole data of spatial distribution, four sections across the entire basin were selected. Figure 2 shows sketch maps of two geological profiles along a northwest-southeast direction (section I-I) and a northeast-southwest direction (section III-III).

Many cities in China are located in various sizes of basins. The city of Fuzhou was selected as an experimental city in China for research and demonstration of the application of earthquake early warning (EEW) and seismic intensity rapid reporting (SIRR) systems, as led by the Seismological Bureau of Fujian Province. Therefore, the parameters of seismic site effects in the Fuzhou basin become an important link in demonstrating the application of the EEW and SIRR systems. Moreover, comprehensive understanding of seismic site effects in Cenozoic basins may be helpful if we consider the Fuzhou basin as a typical example.

\section{Engineering Geological Characteristics of Fuzhou Basin}

The Fuzhou basin is a typical estuarine basin with various types of sediments; the thickness of the covering soil layer is commonly $20-40 \mathrm{~m}$ but reaches $70-90 \mathrm{~m}$ at the deepest part. It is roughly composed of one loose and two soft soil layers and is representatively a coastal soft soil site. The soil layer structure is as follows: the upper part is mainly deposited silt due to transgression and argillaceous fine sand, 


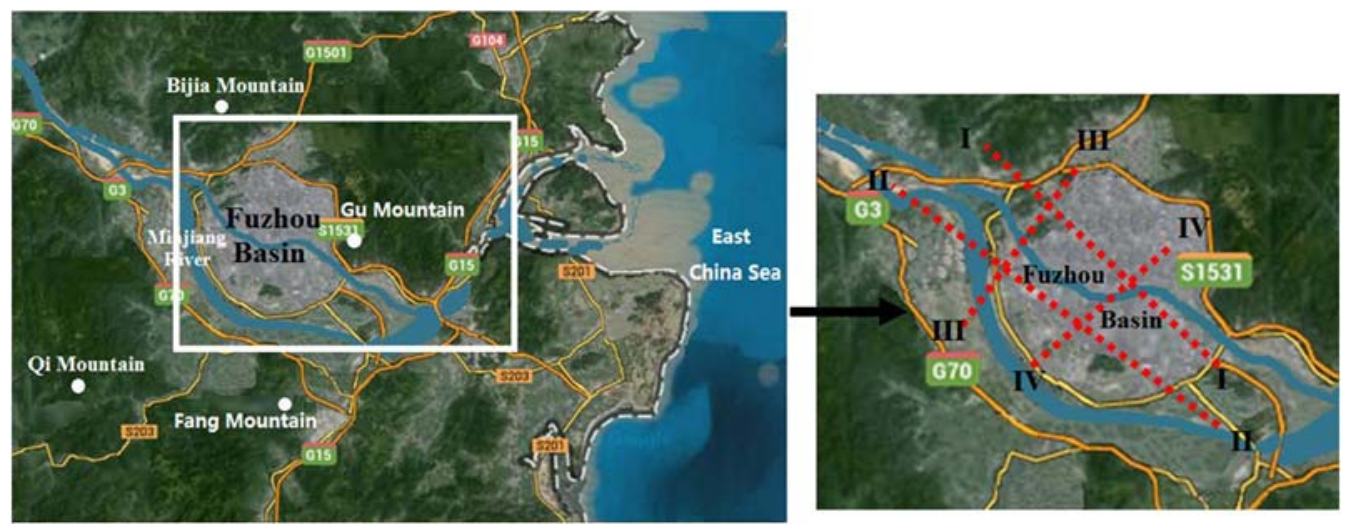

Figure 1. Topography and 2D section locations of the Fuzhou basin. The color version of this figure is available only in the electronic edition.

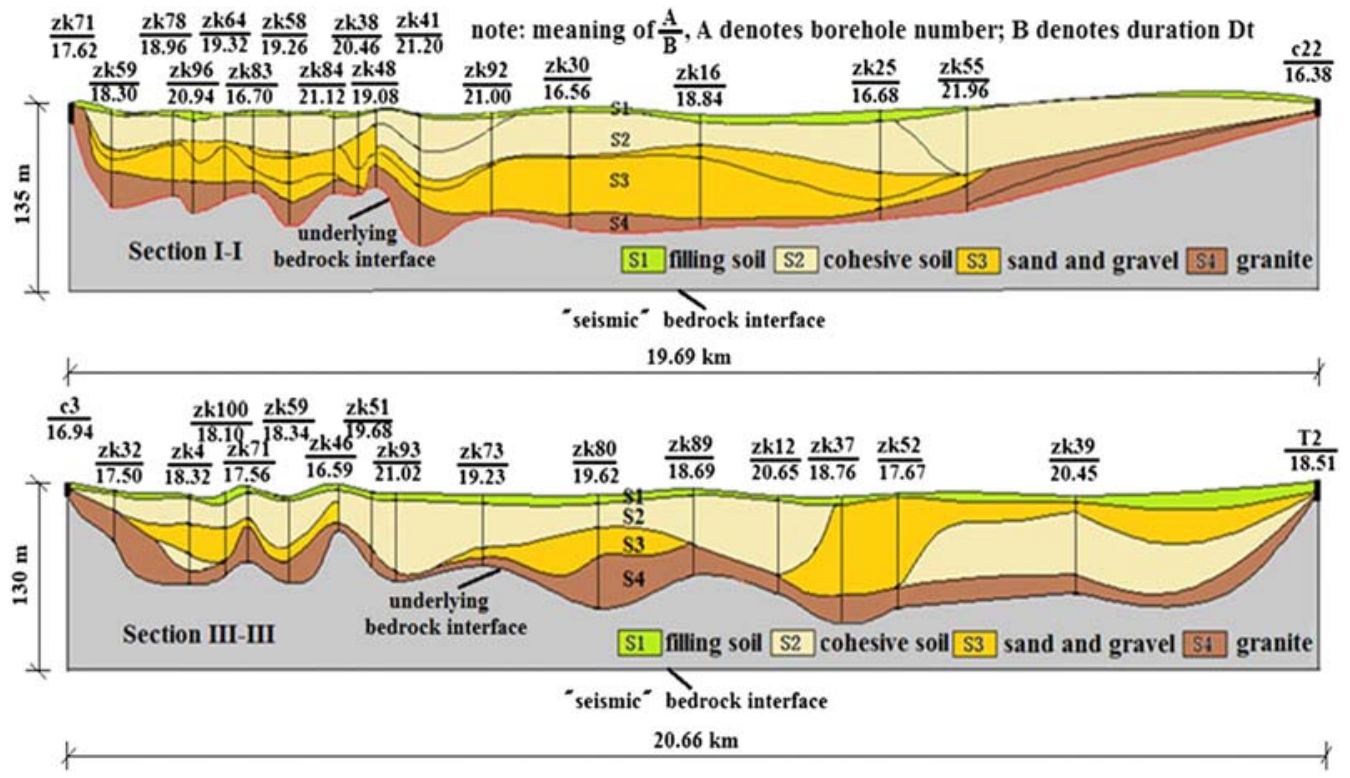

Figure 2. Surface ground-motion durations under input motion w2 for the moderate-level earthquake (MLE) as well as the profiles of Fuzhou basin section I-I. The color version of this figure is available only in the electronic edition.

the middle part is mainly composed of medium-coarse sand, medium-fine sand, mucky soil, and cohesive soils, and the lower part consists of muddy gravel pebbles (rubble) and cohesive soils with gravel. The underlying bedrock is dominated by granites and is known as granite weathering residual soil. This soil is widely distributed in the basin area, and its thickness may be several or even dozens of meters (Zheng, 2013). Table 1 shows detailed information about the soil layer structures, and Figure 3 shows the characteristics of the shear-wave velocity of typical boreholes in the Fuzhou basin. In general, overlying deposition layers in the Fuzhou basin began in the middle stage of the late Pleistocene (approximately 56.5 ka B.P.) and consist of gravel, sand, clay, and silt. Three silt layers were developed: the first two in the middlelate stage of the Holocene (1.44-7.86 ka B.P.) as sediments of the Changle transgression, and the third in the late stage of the late Pleistocene ( $44 \sim 20$ ka B.P.) as sediment of the Fuzhou transgression (Zheng et al., 2005).

The shear-wave velocity with soil depth for each type of soil was analyzed in the Fuzhou basin based on the data from 124 boreholes. Figures 4 and 5 show the shear-wave velocity profile with depth and the equivalent shear-wave velocity contour map, respectively. The equivalent shear-wave velocity for the entire soil deposit was determined, using the method suggested by the China Code for Seismic Design of Buildings GB50011-2010 (Chinese Standard, 2010):

$$
V_{S e}=d_{0} / t
$$

and

$$
t=\sum_{i=1}^{n}\left(d_{i} / V_{S i}\right)
$$




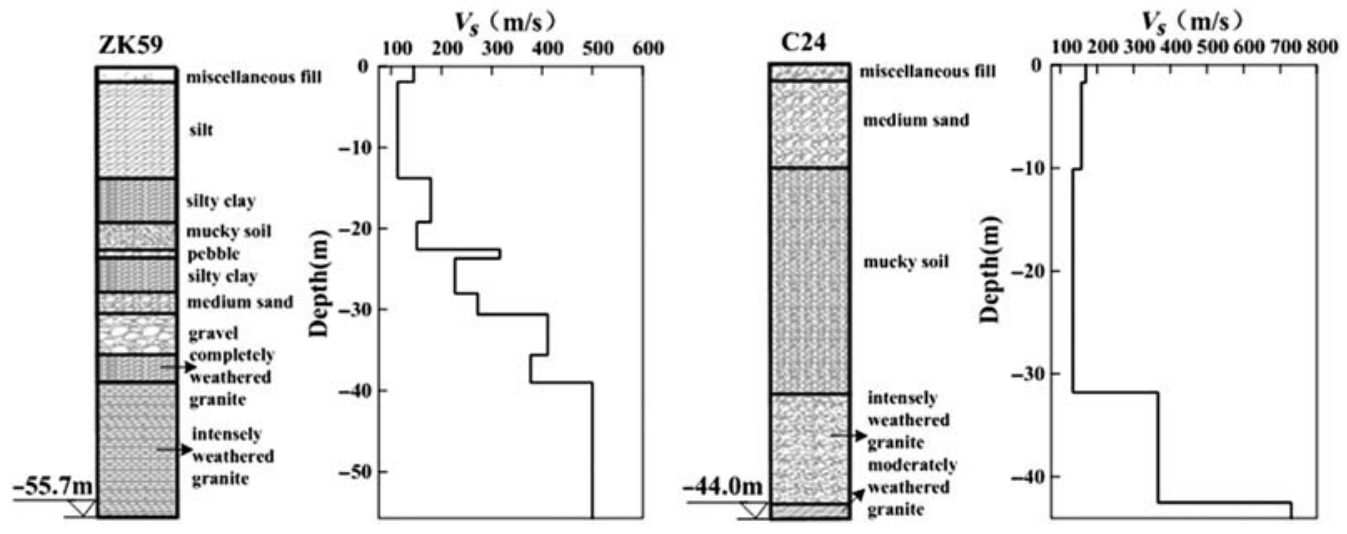

Figure 3. Typical shear-wave velocity profiles and the soil lithology in the Fuzhou basin.

in which $V_{S e}$ is the equivalent shear-wave velocity of the soil layers; $d_{0}$ is the calculation depth, considering the smaller of the two values of either the depth from the ground surface to the underlying bedrock interface or $20 \mathrm{~m}$; $t$ is the shear-wave propagation time from the ground surface to the calculation depth bottom; and $d_{i}$ and $V_{S i}$ denote the thickness and shearwave velocity of the $i$ th layer soil, respectively, in a total of $n$ layers of soil from the ground surface to the underlying bedrock interface.

From Figures 4 and 5, the spatial variation of shear-wave velocity in the Fuzhou basin can be found easily, and a soil classification map based on this (only including site classes II and III) is shown in Figure 6.

\section{Earthquake Environment of Fuzhou Basin}

The distribution of the major faults in the Fuzhou basin is shown in Figure 7 (Wen and Xu, 2003). There has been no destructive earthquake (magnitude $\mathbf{M} \geq 4.75$ ) beneath the city of Fuzhou in recent centuries (information offered by the China Earthquake Administration in 1995), and notably few small earthquakes have been recorded during the past 30 years. Comprehensive explorations show that there is no Holocene active fault among the main faults in the Fuzhou basin, the southeast coast of which is adjacent to the Philippine Sea and the boundary strip area of the Eurasian plate; it is also close to Taiwan, which has a high level of seismic activity. The Fuzhou basin is located in the weakly affected zone of the Taiwan dynamic tentacle in the southeastern coastal areas; however, it is also near the transition boundary region between strongly and weakly affected zones. Moreover, the Fuzhou basin is located in the transition region between $M_{\mathrm{s}} \geq 6.0$ and no $M_{\mathrm{s}} \geq 6.0$ potential earthquake sources. Compared with the Fujian-northeastern Guangdong region, where strong earthquakes have occurred, the most recent time when the main fractures of the Fuzhou basin and its adjacent regions were active was a relatively long time ago, and the low-velocity layer in the crust is not significant. However, the seismogenic potential for a medium-strong destructive earthquake in the Fuzhou basin cannot be ruled out. The maximum possible earthquake magnitude has been estimated as $M_{\mathrm{s}} 6.0$ (Wen and $\mathrm{Xu}, 2003$ ).

\section{Nonlinear Cyclic Behaviors of Soils}

The stress-strain relationship of soils shows nonlinear characteristics, hysteretic quality, and accumulative deformation under cyclic loadings. The soil constitutive model

Table 1

The Main Soil Layer Structures of Fuzhou Basin

\begin{tabular}{|c|c|c|c|c|}
\hline Soil Layer Structures & \multicolumn{2}{|c|}{ Main Soil Types } & Thickness & Characteristics \\
\hline \multirow[t]{2}{*}{ Upper layer } & Upper segment & Mainly clay & Approximately $2 \mathrm{~m}$ & Surface soil covered by artificial miscellaneous fills \\
\hline & Lower segment & $\begin{array}{l}\text { Deposited silt, } \\
\quad \text { argillaceous fine sand }\end{array}$ & $20-30 \mathrm{~m}$ & Highly compressed soft silt \\
\hline Middle layer & \multicolumn{2}{|c|}{$\begin{array}{l}\text { Mainly medium-coarse sand, medium-fine } \\
\text { sand, mucky soil, and cohesive soils }\end{array}$} & $30-35 \mathrm{~m}$ & $\begin{array}{l}\text { Soft soil layer with varying thicknesses are widely } \\
\text { distributed in the medium-coarse sand and } \\
\text { medium-fine sand layers }\end{array}$ \\
\hline Lower layer & \multicolumn{2}{|c|}{$\begin{array}{l}\text { Mainly muddy gravel pebble (rubble) and } \\
\text { cohesive soils with gravel }\end{array}$} & $20-30 \mathrm{~m}$ & $\begin{array}{l}\text { The strata structure changes significantly in both } \\
\text { the horizontal and vertical directions due to } \\
\text { multiple historical changes of the Minjiang } \\
\text { paleochannel }\end{array}$ \\
\hline $\begin{array}{l}\text { Underlying bedrock } \\
\text { layer }\end{array}$ & \multicolumn{2}{|c|}{$\begin{array}{l}\text { Dominated by granites named granite } \\
\text { weathering residual soil }\end{array}$} & $\begin{array}{l}\text { Several meters, even } \\
\text { dozens of meters }\end{array}$ & $\begin{array}{l}\text { Remained after long-term physical and chemical } \\
\text { weathering and is widely distributed }\end{array}$ \\
\hline
\end{tabular}


(a)

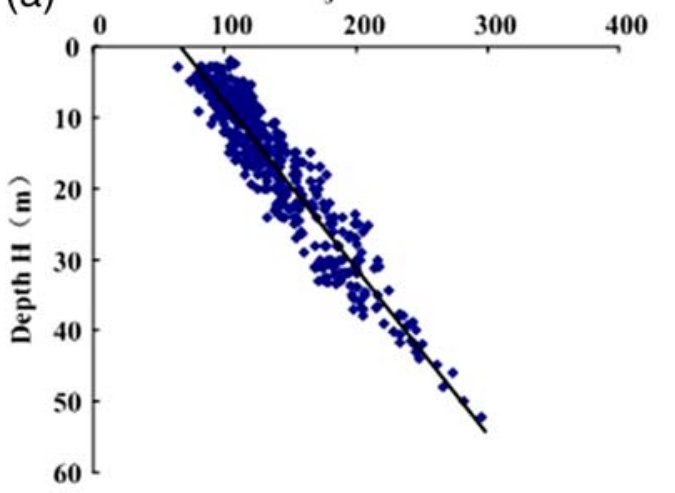

(c)

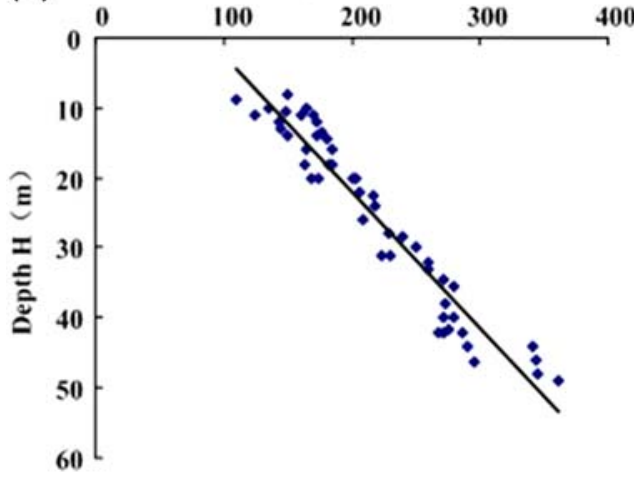

(b)

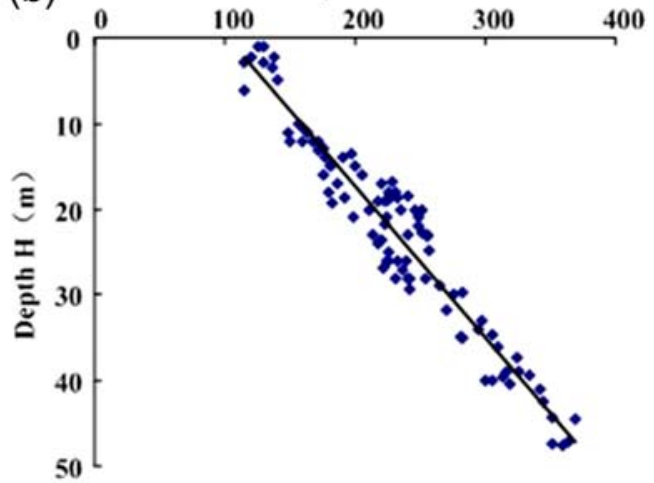

(d)

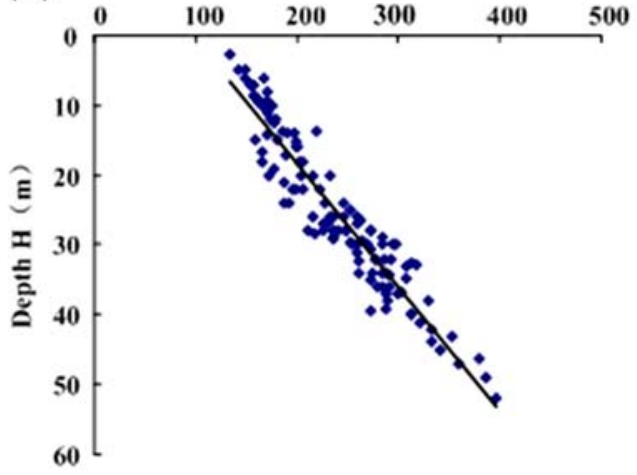

Figure 4. Fitting curves of shear-wave velocities with soil depth in the Fuzhou basin: (a) mucky soil, (b) silty clay, (c) fine sand, and (d) medium-coarse sand. The color version of this figure is available only in the electronic edition.

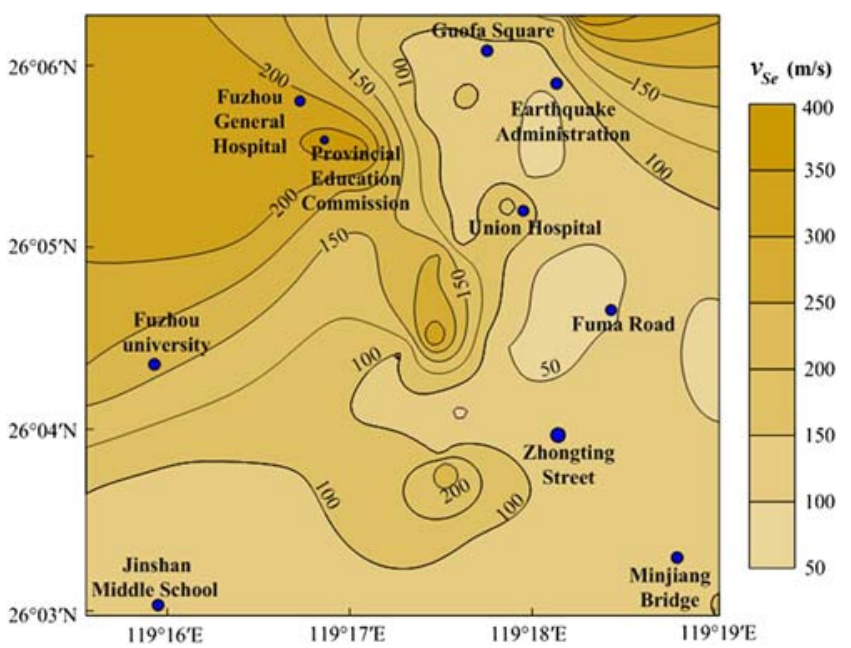

Figure 5. Contour map of the equivalent shear-wave velocity of the soil layers for the Fuzhou basin. The color version of this figure is available only in the electronic edition.

utilized in this numerical study is the modified MairtinSeed-Davidenkov viscoelastic model (Chen and Zhuang, 2005), which is based on the Davidenkov skeleton curve of the 1D dynamic stress-strain relationship. The dynamic shear-stress-strain curves were constructed using Masing rules. The Davidenkov skeleton curve was corrected using sectional functions, and the upper limit of the failure shear-

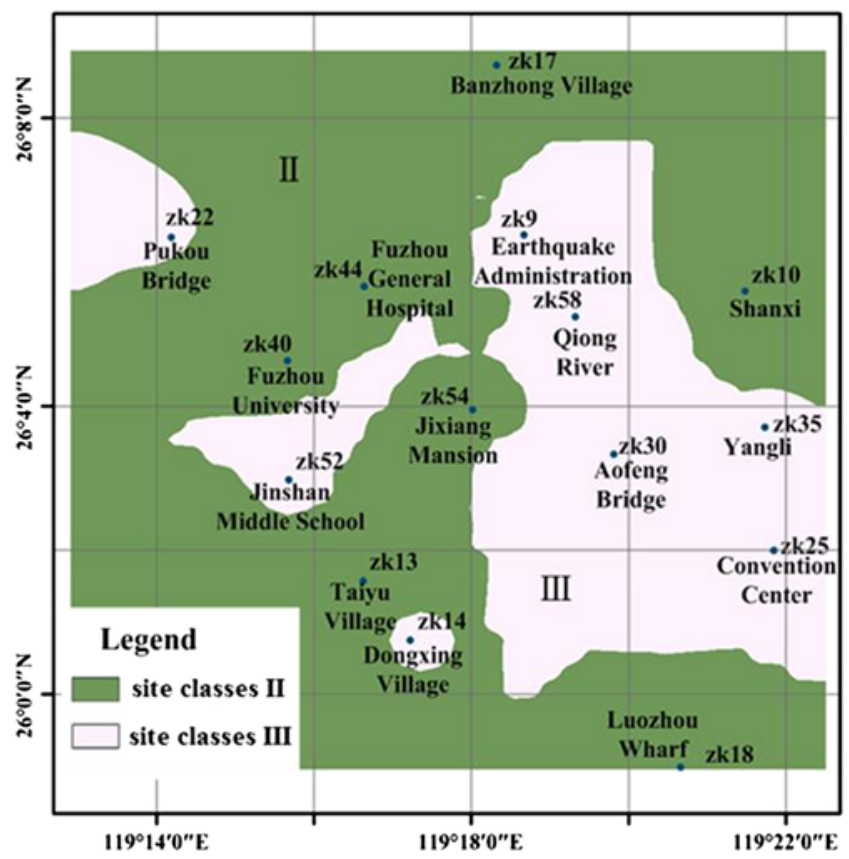

Figure 6. Soil classification map of the Fuzhou basin. The color version of this figure is available only in the electronic edition.

strain amplitude was used as the sectional point. The modified skeleton curve could approach the upper limit of failure shear stress when shear-strain amplitude was observed to 


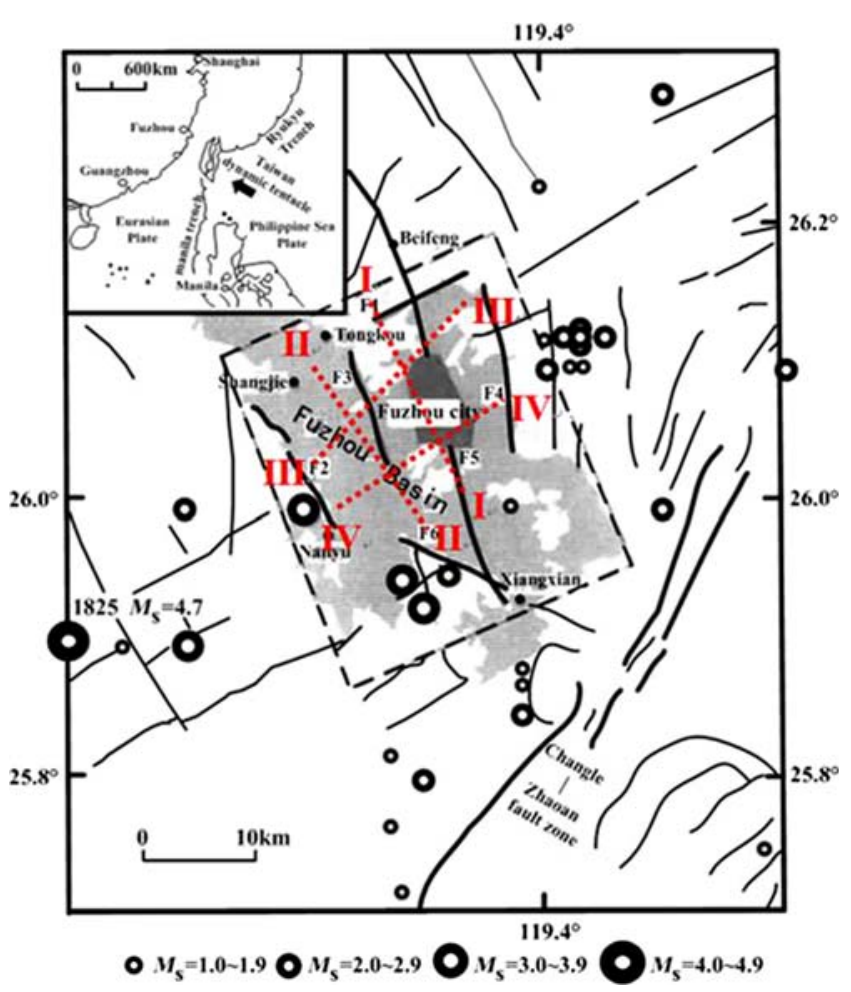

Figure 7. Sketch maps of the main faults and the epicentral distribution of earthquakes since 1971 in the Fuzhou basin (shaded area) and its neighboring regions (Wen and $\mathrm{Xu}, 2003$ ). The four dotted lines are the section lines of the $2 \mathrm{D}$ site response analysis in this article. The box indicated by dashed lines represents the detailed exploration area, and the fault lines in bold denote the middlelate Pleistocene fault; the thin fault lines denote the active fault before the middle Pleistocene; open circles represent the epicenters of historical destructive earthquakes; F1-F6 represent the serial numbers of the main faults; the inset at the top left corner indicates the location of the Fuzhou basin in the areas of regional plate tectonic activity. The color version of this figure is available only in the electronic edition.

approach infinity. The hysteretic curve of the modified shear stress and strain relationship is shown in Figure 8.

The Davidenkov skeleton curve put forward by Martin and Seed (1982) is

$$
\tau(\gamma)=G \times \gamma=G_{\max } \times \gamma \times[1-H(\gamma)],
$$

in which

$$
H(\gamma)=\left\{\frac{\left(\gamma / \gamma_{0}\right)^{2 B}}{\left[1+\left(\gamma / \gamma_{0}\right)\right]^{2 B}}\right\}^{A}
$$

The shear stress and strain relationship of soils should be $\gamma \rightarrow \infty, \tau(\gamma) \rightarrow \tau_{\text {ult }}$ (the upper limit of the shear stress); however, equations (3) and (4) indicate that $\gamma \rightarrow \infty$, $\tau(\gamma) \rightarrow \infty$, which is not consistent with the actual soil behavior. There would be an upper-limit value of strain $\left(\gamma_{\text {ult }}\right)$ for each type of soil: when the shear-strain amplitude $(\gamma)$ exceeds $\gamma_{\text {ult }}$, which is in a critical state of failure, any further increase of $\gamma$ may not induce an increase in shear stress,

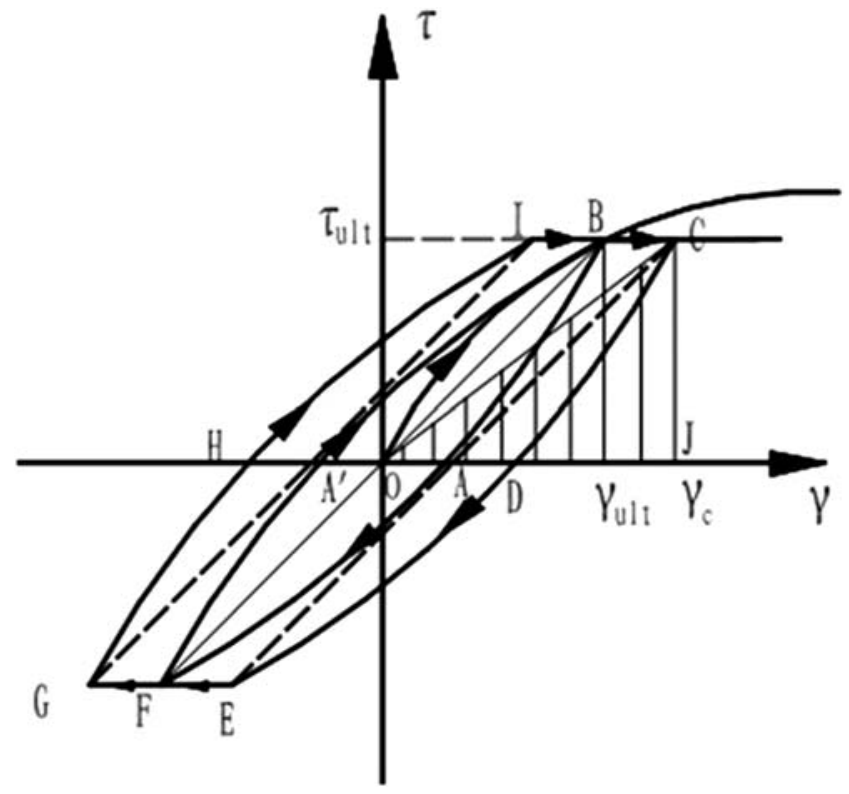

Figure 8. Hysteretic loop of the shear stress-strain of the modified Davikendov model for one cycle of loading.

or there could even be a decreasing trend in shear stress. Therefore, the piecewise function method is used to describe the skeleton curve in the article, and the Davidenkov model was modified as follows (Chen and Zhuang, 2005):

$$
\tau(\gamma)= \begin{cases}G_{\max } \times \gamma \times[1-H(\gamma)] & \gamma_{c} \leq \gamma_{\mathrm{ult}} \\ G_{\max } \times \gamma_{\mathrm{ult}} \times\left[1-H\left(\gamma_{\mathrm{ult}}\right)\right] & \gamma_{c}>\gamma_{\mathrm{ult}}\end{cases}
$$

and

$$
\tau_{\mathrm{ult}}=G_{\mathrm{max}} \times \gamma_{\mathrm{ult}} \times\left[1-H\left(\gamma_{\mathrm{ult}}\right)\right] .
$$

The upper-limit value of the shear strain of the modified Mairtin-Seed-Davidenkov constitutive model has a definite physical meaning for soils, and the parameters of the constitutive model can be obtained easily by general cyclic loading. Because of this property, by means of the VUMAT connector of the ABAQUS software, the subroutine code of the above soil constitutive model has been embedded using FORTRAN (Chen et al., 2011). Hence, user-defined materials may be realized by employing the constitutive model subroutine.

From the ground surface to the underlying bedrock interface, miscellaneous fill, clay, mucky silty clay, silty clay, fine sand, medium-coarse sand, gravel, etc., exist. According to the borehole data, as well as the resonant column test results for typical soil samples of the Fuzhou basin, Figure 9 and Table 2 show the normalized shear modulus and the damping ratio curves and parameter values for typical soils tested by free vibration column apparatus, respectively.

\section{Seismic Bedrock Motions}

Taking the interface of elastic homogeneous bedrock and nonhomogeneous weathered rock or soil as the seismic 


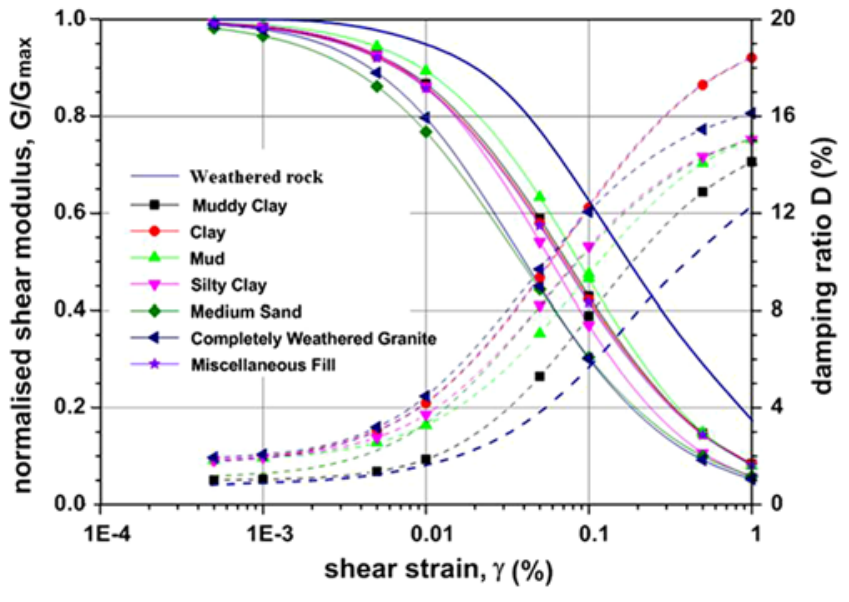

Figure 9. Modulus degradation and damping ratio curves of Fuzhou basin soils. The color version of this figure is available only in the electronic edition.

bedrock interface of input motions, an input of seismic bedrock motions can be seen as $S$-wave propagation in a vertical direction. According to the borehole data for the Fuzhou basin, most boreholes are deep enough to reveal the underlying bedrock, and the weathered rock layers with shear-wave velocity larger than $500 \mathrm{~m} / \mathrm{s}$ were selected as the seismic bedrock interface of the input motions. It is noteworthy that the upper segment of bedrock of most boreholes consists of strongly weathered rock layers.

Because of a lack of actual ground-motion records in southeast China and especially because no destructive earthquake has occurred beneath the city of Fuzhou in recent centuries, artificial earthquake acceleration time histories were adopted in this article. According to the China Code for Evaluation of Seismic Safety for Engineering Sites GB17741-2005 (Chinese Standard, 2005), the peak acceler- ations and target acceleration response spectra of bedrock motions with $63 \%, 10 \%$, and $2 \%$ probability of exceedance in 50 years for the Fuzhou basin (see Fig. 10) were obtained using the China probabilistic seismic-hazard analysis method. The peak accelerations were approximately $50 \mathrm{~cm} / \mathrm{s}^{2}$, $100 \mathrm{~cm} / \mathrm{s}^{2}$, and $150 \mathrm{~cm} / \mathrm{s}^{2}$, respectively, which were defined as a low-level earthquake (LLE), a moderate-level earthquake (MLE), and a high-level earthquake (HLE), respectively. A method adjusting both the phase and spectral values $(\mathrm{Hu}$ and $\mathrm{He}, 1986)$ was used to synthesize the artificial earthquake waves (w1-w6) compatible with the peak accelerations and target spectra for bedrock motions in this article. Six artificial waves with different frequency characteristics for the LLE, MLE, and HLE cases were synthesized and used as input motions of the seismic bedrock interface (abbreviated as w1, w2, w3, w4, w5, and w6, respectively). Figures 11-12 show acceleration time histories and power spectra for the six artificial earthquake waves. In addition, the Kobe wave seismic record recorded at station KJMA, which had $18.27 \mathrm{~km}$ of epicentral distance in the 1995 Kobe earthquake, Japan, was also selected as the input motion. The PGA of the original Kobe record $\left(85 \mathrm{~cm} / \mathrm{s}^{2}\right)$ was adjusted to be equal to $50 \mathrm{~cm} / \mathrm{s}^{2}, 100 \mathrm{~cm} / \mathrm{s}^{2}$, and $150 \mathrm{~cm} / \mathrm{s}^{2}$ for an LLE, MLE, and HLE, respectively, by adjusting the peak acceleration of the original Kobe record. Figure 13 shows the acceleration time history and the power spectrum resulting from adjusting the Kobe record for the MLE case; the Kobe wave had a relatively abundant low-frequency component and roughly equivalent energy to any of the six artificial waves.

\section{Artificial Boundary Conditions}

It may be a key problem to simulate the unbounded site for seismic site effect evaluation. In this article, based on the specific features of the 2D sections of the Fuzhou basin, 2D

Table 2

Values of the Normalized Shear Modulus and the Damping Ratio of Soils for Fuzhou Basin

\begin{tabular}{|c|c|c|c|c|c|c|c|c|c|}
\hline \multirow[b]{2}{*}{ Soils } & \multirow[b]{2}{*}{ Parameters* } & \multicolumn{8}{|c|}{ Shear-Strain Amplitude $\left(\gamma / 10^{-4}\right)$} \\
\hline & & 0.05 & 0.1 & 0.5 & 1 & 5 & 10 & 50 & 100 \\
\hline \multirow[t]{2}{*}{ Miscellaneous fill } & $G / G_{\max }$ & 0.991 & 0.982 & 0.921 & 0.858 & 0.574 & 0.416 & 0.143 & 0.082 \\
\hline & $D(\%)$ & 1.77 & 1.93 & 3.05 & 4.21 & 9.43 & 12.32 & 17.33 & 18.44 \\
\hline \multirow[t]{2}{*}{ Muddy clay } & $G / G_{\max }$ & 0.992 & 0.984 & 0.927 & 0.867 & 0.588 & 0.429 & 0.147 & 0.084 \\
\hline & $D(\%)$ & 1.01 & 1.04 & 1.37 & 1.87 & 5.29 & 7.78 & 12.88 & 14.12 \\
\hline \multirow[t]{2}{*}{ Clay } & $G / G_{\max }$ & 0.991 & 0.983 & 0.923 & 0.861 & 0.580 & 0.422 & 0.146 & 0.084 \\
\hline & $D(\%)$ & 1.82 & 1.97 & 3.06 & 4.19 & 9.35 & 12.23 & 17.29 & 18.42 \\
\hline \multirow[t]{2}{*}{ Mud } & $G / G_{\max }$ & 0.994 & 0.988 & 0.944 & 0.894 & 0.633 & 0.465 & 0.149 & 0.080 \\
\hline & $D(\%)$ & 1.84 & 1.93 & 2.56 & 3.28 & 7.06 & 9.50 & 14.07 & 15.06 \\
\hline \multirow[t]{2}{*}{ Silty clay } & $G / G_{\max }$ & 0.993 & 0.986 & 0.927 & 0.861 & 0.541 & 0.370 & 0.107 & 0.057 \\
\hline & $D(\%)$ & 1.84 & 1.95 & 2.77 & 3.72 & 8.21 & 10.63 & 14.34 & 15.05 \\
\hline \multirow[t]{2}{*}{ Medium sand } & $G / G_{\max }$ & 0.982 & 0.966 & 0.862 & 0.768 & 0.443 & 0.304 & 0.100 & 0.058 \\
\hline & $D(\%)$ & 1.18 & 1.29 & 2.25 & 3.36 & 8.16 & 10.53 & 14.28 & 15.08 \\
\hline \multirow[t]{2}{*}{ Complete weathered granite } & $G / G_{\max }$ & 0.990 & 0.979 & 0.890 & 0.797 & 0.450 & 0.301 & 0.092 & 0.052 \\
\hline & $D(\%)$ & 1.94 & 2.07 & 3.19 & 4.47 & 9.69 & 12.06 & 15.46 & 16.12 \\
\hline \multirow[t]{2}{*}{ Underlying weathered rock } & $G / G_{\max }$ & 0.999 & 0.998 & 0.992 & 0.956 & 0.859 & 0.642 & 0.396 & 0.193 \\
\hline & $D(\%)$ & 0.98 & 1.02 & 1.12 & 1.65 & 2.73 & 5.43 & 9.76 & 12.25 \\
\hline
\end{tabular}

$* G / G_{\max }$ denotes the ratio of dynamic shear modulus versus maximum shear modulus; $D(\%)$ denotes the damping ratio $(\%)$. 


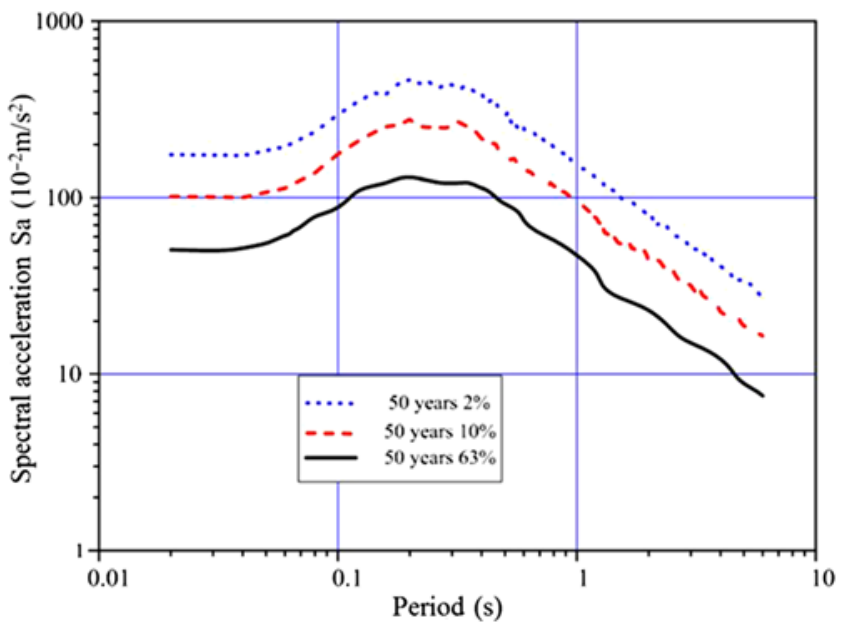

Figure 10. Bedrock target acceleration response spectra of a site in the Fuzhou basin for $63 \%, 10 \%$, and $2 \%$ probability of exceedance in 50 years. The color version of this figure is available only in the electronic edition.

viscoelastic artificial boundaries are adopted, which involve dashpots attached independently to the boundary in the normal and shear directions. These viscosity terms are not introduced into the equations of motion of the grid points lying on the boundary, but the normal and shear tractions are calculated and applied at every time step in the same way boundary loads are applied. As a result, allowing seismicwave energy radiation propagation toward the far-field sites prevents the reflection of outward propagating waves back into the model or nearly approximates nonreflective waves on the truncated boundary.

The spring and damping coefficients of the artificial boundary equivalent physical system are calculated in two directions (Liu et al., 2006):

$$
\text { Tangential direction : } K_{\mathrm{BT}}=\alpha_{\mathrm{T}} \frac{G}{R}, \quad C_{\mathrm{BT}}=\rho C_{S}
$$

$$
\text { Normal direction : } K_{\mathrm{BN}}=\alpha_{\mathrm{N}} \frac{G}{R}, \quad C_{\mathrm{BN}}=\rho C_{P} \text {, }
$$

where $R$ is the distance from wave source to the artificial boundary; $C_{S}$ and $C_{P}$ are the shear-wave and compression-wave velocity, respectively; $G$ is the shear modulus; $\rho$ is the mass density; and $\alpha_{\mathrm{T}}$ and $\alpha_{\mathrm{N}}$ are the parameters of the viscoelastic artificial boundary in the tangential and normal direction, respectively. Calculation results ( $\mathrm{Liu}$ and $\mathrm{Li}$, 2005) indicated that these two parameters were robust, in (a)

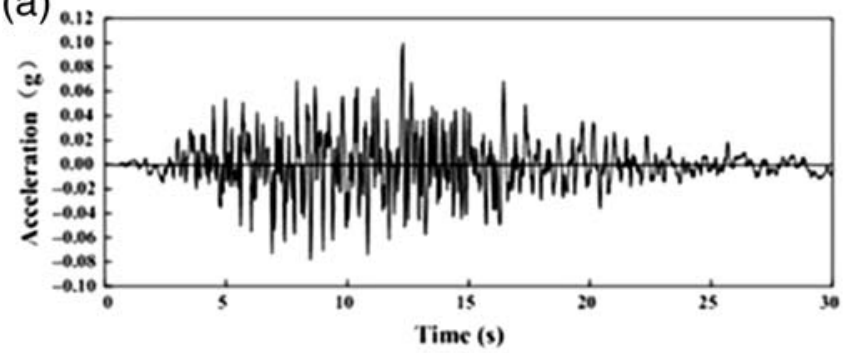

(c)

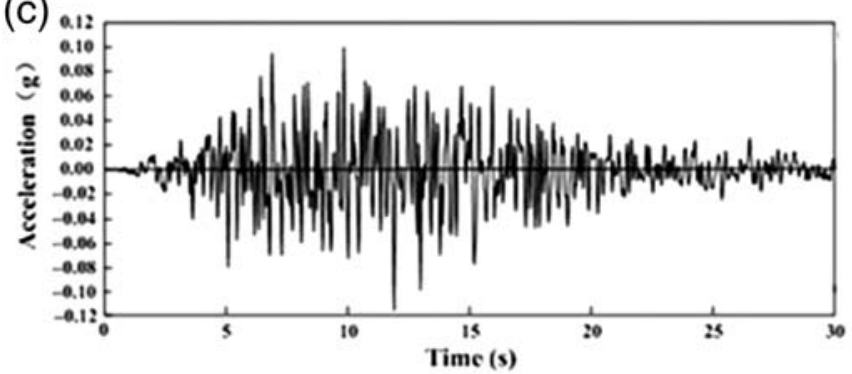

(e)

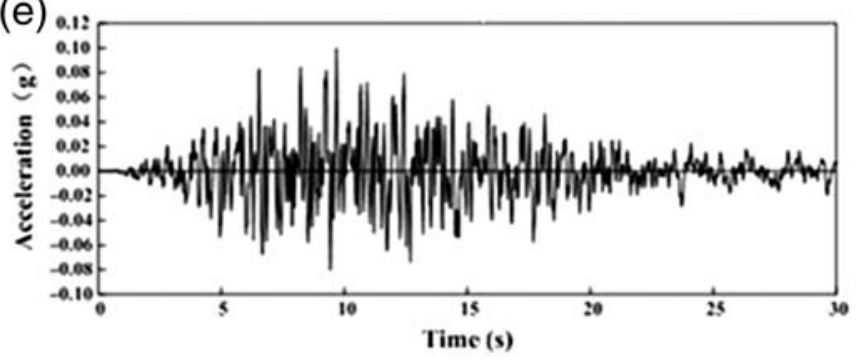

(b)

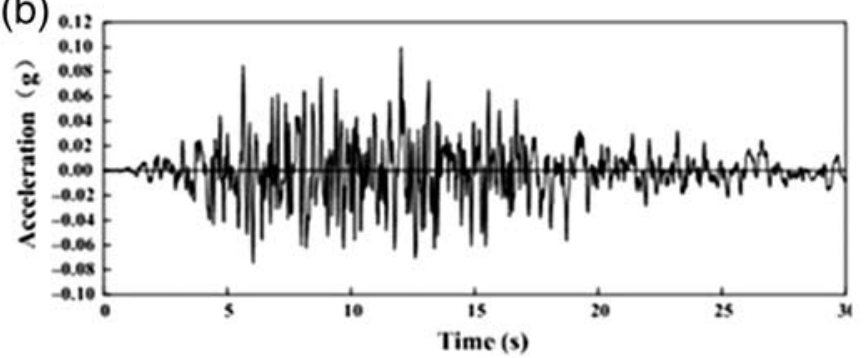

(d)

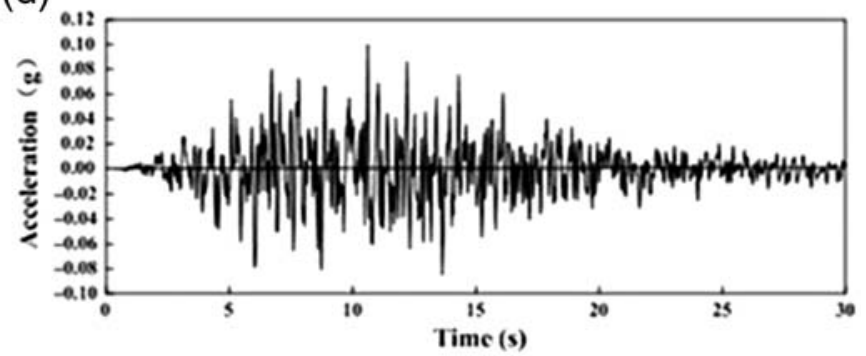

(f)

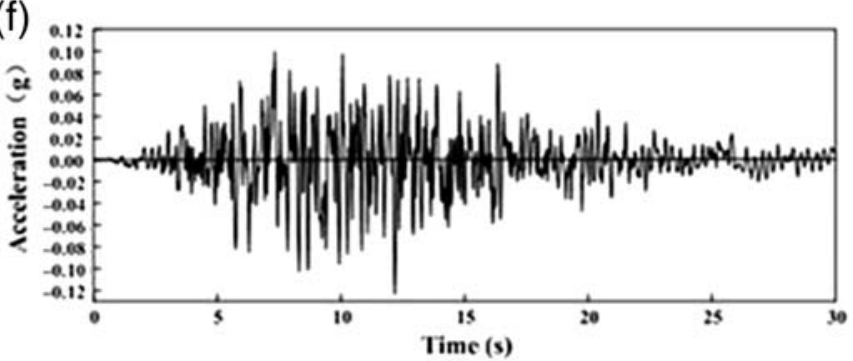

Figure 11. Acceleration time histories of the artificial bedrock motions w1-w6 for an MLE: (a) w1; (b) w2; (c) w3; (d) w4; (e) w5; and (f) w6. 
(a)

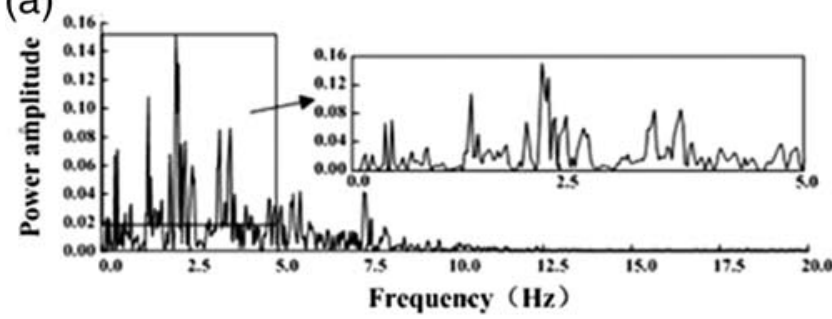

(c)

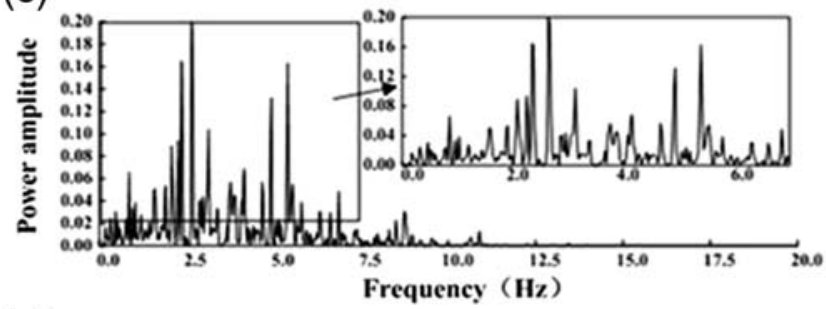

(e)

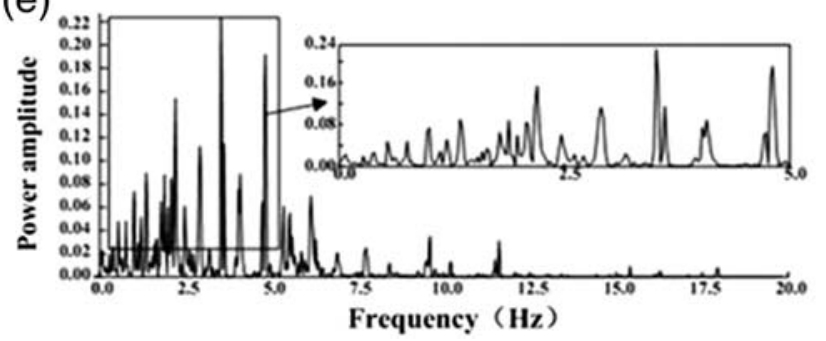

(b)

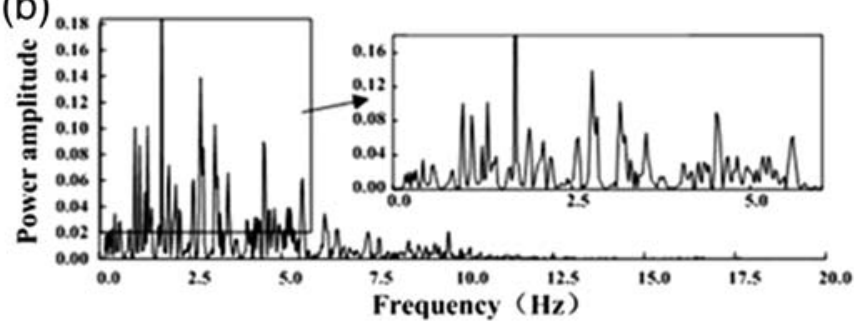

(d)

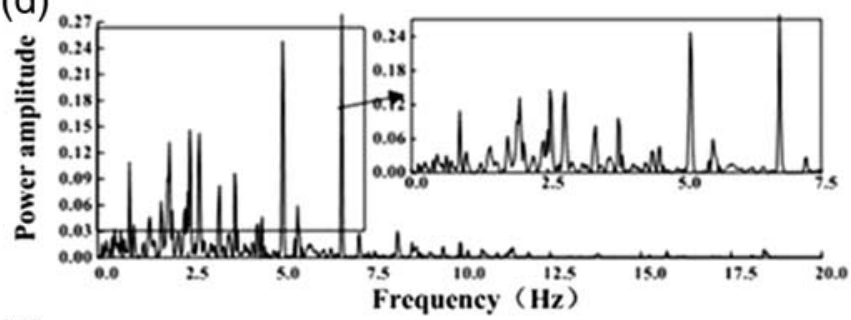

(f)

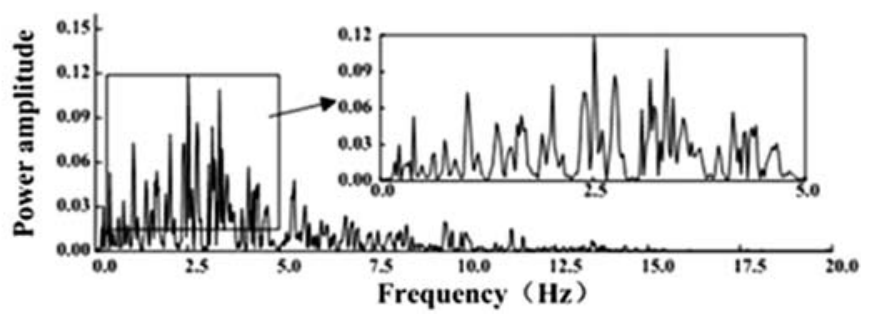

Figure 12. Power spectra of the artificial bedrock motions w1-w6 for an MLE: (a) w1; (b) w2; (c) w3; (d) w4; (e) w5; and (f) w6.

(a)

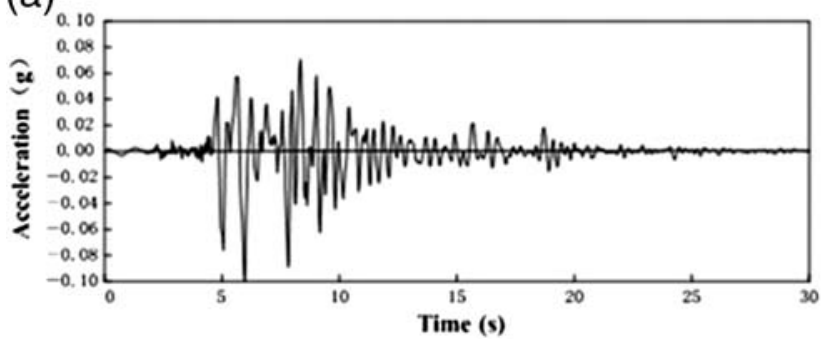

(b)

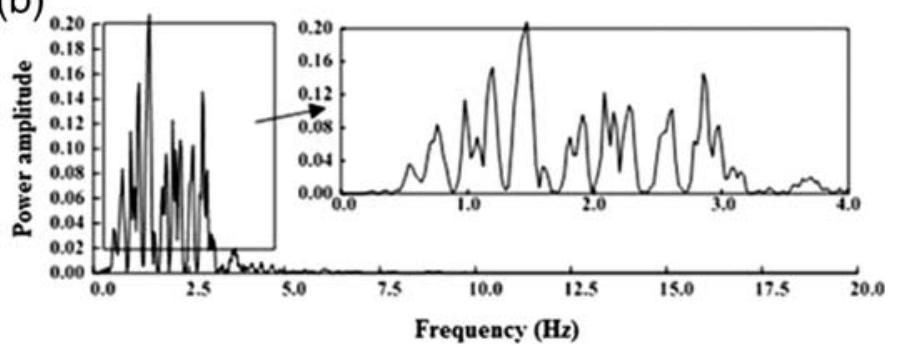

Figure 13. Acceleration time history and power spectrum of the adjusted Kobe record for an MLE: (a) acceleration time histories and (b) power spectra.

other words, they can be valued within a certain range without affecting the calculation accuracy. The recommended interval for $\alpha_{\mathrm{T}}$ is $[0.35,0.65]$ and for $\alpha_{\mathrm{N}}$ is $[0.8,1.2]$. In this article, $\alpha_{\mathrm{T}}$ is 0.5 , and $\alpha_{\mathrm{N}}$ is 1.0 .

These boundary conditions can not only overcome the low-frequency instability problems of the viscous boundary, but also simulate the elastic recovery performance of the foundation, thereby conferring good stability.

\section{Element Type}

It is necessary to consider the changes of shear-wave velocity with soil depth. The finite-element grid maximum size at the direction of wave propagation is set as $1 / 8-1 /$ 12 of the minimum wavelength, corresponding to the cutoff frequency, which is set as $20 \mathrm{~Hz}$ in this article. The vertical element size is set to be $1-1.2 \mathrm{~m}$, and the horizontal element size is set as $3-5 \mathrm{~m}$. To ensure computational accuracy and reduce computation time, quadrilateral elements are mainly adopted and triangular elements are also used.

\section{Large-Scale 2D Simulation Approach}

Based on the explicit finite-element method and parallel computing technology (Chen et al., 2011), the seismic site effect of the Fuzhou basin was simulated, and the equations 

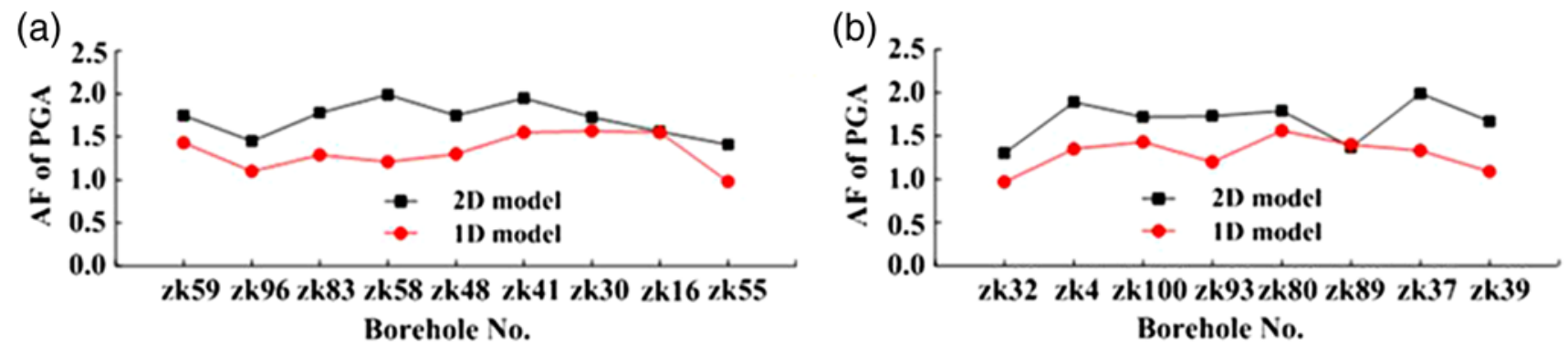

Figure 14. Comparison between the $1 \mathrm{D}$ and $2 \mathrm{D}$ results of peak ground acceleration (PGA) at the basin surface for seismic bedrock motion w1 corresponding to the MLE case: (a) section I-I and (b) section III-III. The color version of this figure is available only in the electronic edition.

of motion were integrated with respect to time by the central difference method in ABAQUS/Explicit. The solution of the motion equation is expressed as

$$
\ddot{\mathbf{u}}^{(i)}=\mathbf{M}^{-1} \times\left(\mathbf{F}^{(i)}-\mathbf{I}^{(i)}\right)
$$

in which $\mathbf{M}$ is the lumped mass matrix, $\mathbf{F}$ is the applied load vector, and $\mathbf{I}$ is the vector of unit internal force.

As there is no need to compute the inverse of the stiffness matrix when using the explicit algorithm method, using explicit algorithm method for models with high degrees of freedom for numerical calculations confers a great advantage. In addition, another advantage is that the viscoelastic artificial boundary set in ABAQUS may well simulate radiation damping in the semi-infinite site.

\section{The 1D Model for Calibration and Supplement}

Different authors have applied 1D models to evaluate site effects at Mexico City for the vertical propagation of the $S H$ wave. For example, Seed et al. (1988) performed a parametric study with $S H A K E$ software for a $1 \mathrm{D}$ model. This software is one of the most commonly used tools in site response analysis for a system of homogenous, viscoelastic layers of infinite horizontal extent, as it has been shown to produce acceptable results when the site is subjected to small soil shear strains (less than $\sim 1 \%$ ) and modest PGAs (less than $\sim 0.3 g$ ). Therefore, the Proshake software, which was originally developed from $S H A K E$, was chosen as the study tool in this article.

In the equivalent linear approach, linear analyses with soil properties are performed and iteratively adjusted to be consistent with an effective level of shear strain induced in the soil. As the level of shear strain increases, the secant shear modulus decreases. The relationship between the secant shear modulus and the shear-strain amplitude can be characterized by means of a normalized shear modulus degradation curve, which has the modulus ratio on the ordinate and log (shear strain) on the abscissa, and has been well established for many soils. Proshake has a library of modulus degradation relationships that can be selected in the Input Manager.
To some extent, the 1D results from Proshake were used as a reference for verification of the $2 \mathrm{D}$ results and as a supplement for the analysis of the seismic site response in this article. Zhan (2012) noted that the Proshake and ABAQUS results show similar characteristics for the seismic site effect in regions with a flat underlying bedrock interface and horizontal soil layers, especially for the PGA of input motions less than $0.3 g$. Hence for calibration purposes, surface ground motion at some locations of the basin was investigated by comparing the results of the $1 \mathrm{D}$ equivalent linear model performed in the frequency domain with the results of the 2D nonlinear model performed in the time domain. The results are presented in Figure 14 and indicate that almost all PGA amplification factors for all borehole surfaces (abbreviated as $\mathrm{AF}_{\mathrm{PGA}}$ ) were expressed as consistent differences for MLE cases and that the results of the $2 \mathrm{D}$ analyses either were higher or close to those for the 1D analysis or showed a more significant amplification effect than the 1D results. Recently, a number of studies, made possible by the availability of a large amount of quality strong-motion data, indicated the nonlinearity of soils, which is influenced by topography and soil spatial variation (Rubinstein, 2011; Lanzo and Pagliaroli, 2012). As a result, both the topographic inequality and the lateral inhomogeneity of soils should be taken into consideration, especially for large-scale complex sites. If this is done, 1D analysis is not more applicable for those complex site problems. Moreover, both theoretical and empirical studies have indicated that the 2D nonlinear analysis is reliable for seismic site effects; it reflects the influence of terrain features and soil lateral heterogeneity.

\section{Analysis of Seismic Site Effects of Fuzhou Basin}

\section{Spatial Variation of PGA}

Figure 15 shows the PGA amplification factors in different locations of the basin surface, which were normalized by the PGA of seismic bedrock motions. Site amplification effects of surface ground motions are easily found and are especially significant in some locations, such as the PGA of the borehole zk58 and zk41 surfaces in Figure 15. The PGA amplification factors in the depression areas of the underlying bedrock interface shape seem larger than those 

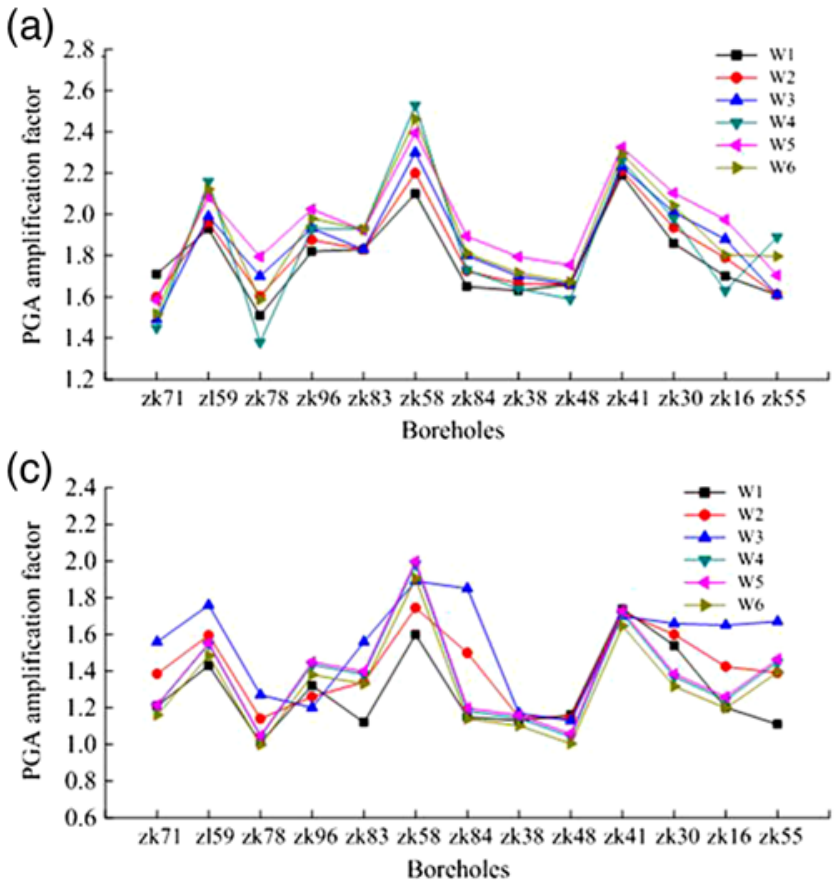
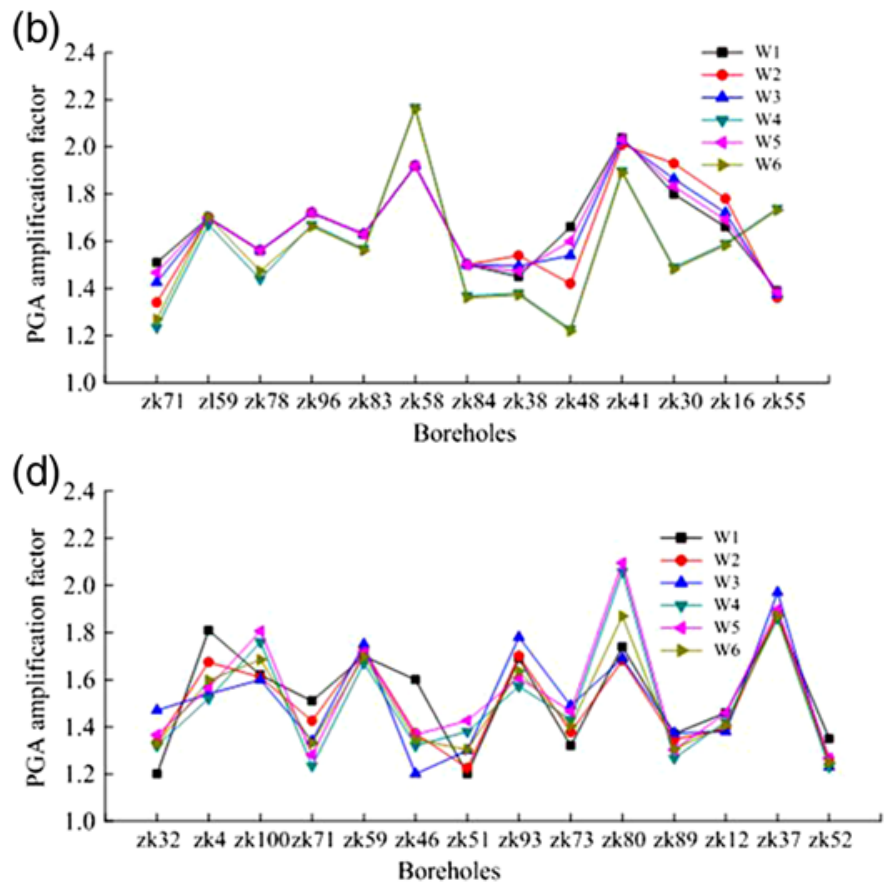

Figure 15. PGA amplification factors at different borehole surfaces for different seismic bedrock motions: (a) section I-I for the low-level earthquake (LLE) case; (b) section I-I for the MLE case; (c) section I-I for the high-level earthquake (HLE) case; and (d) section III-III for the MLE case. The color version of this figure is available only in the electronic edition.

for other locations. There are similar PGA amplification phenomena in the same section for different seismic bedrock motion levels.

The Fuzhou basin is typically a Cenozoic fault basin, which is also adjacent to the gulf. There are obvious upheaval and depression areas of the underlying bedrock interface shape where the variation gradient of both the shearwave velocity and depth are greater, hence surface waves easily converge during seismic-wave propagation The propagating seismic-wave energy may be focused in some fluctuating areas (upheaval and depression areas), which makes the PGA amplification of surface ground motion significant. This type of focusing effect was also found in the direction of depth in some locations.

Figure 16 shows spatial variation characteristics of the PGA amplification factors varying with soil depth and lateral direction of section I-I and section III-III for different seismic bedrock motion levels. (1) Borehole zk58 and zk41 locations in Figure 16a correspond to the obvious depression areas of the underlying bedrock interface, where the PGAs have nonmonotonic decreasing characteristics with soil depth and a greater focusing effect of some shallow soil layers. It indicates that there is the focusing effect of seismic-wave propagation in the depth direction; for example, the PGA amplification effect of soils approximately $5 \mathrm{~m}$ below the surface (Fig. 16a) and $10 \mathrm{~m}$ below the surface (Fig. 16d) are relatively larger than that of other soil depth. Figure 17 likewise shows the focusing effect with soil depth. The shape of the depression underlying the bedrock interface probably induces the focusing of seismic-wave propagating energy, and reflection and refraction occur during the seismic-wave propagation among the heterogeneous soil layers; thus, there is constructive interference, which makes the seismic wave gather in an appropriate depth. In addition, there are always some deeper soft soil layers, which may be another important cause of the focusing effect of PGA amplification with depth. (2) The focusing effect of PGA amplification is not so obvious in those areas of flat underlying bedrock interface or smooth terrain, and it turns out that PGA basically decreases monotonically with soil depth except for abrupt increases at a few particular soil depths. (3) The PGA of the regions close to the basin edge is larger than that in flat regions; for example, the borehole zk59 and zk32 locations near the basin edge in Figure 16a,d, whose PGA at different depths seems relatively larger. It may be that when the seismic body wave is transmitted along the edge of the basin, it generates a surface wave or a diffraction wave that interacts with the incident seismic waves, leading to constructive interference, and causes repeated reflection of seismic waves in the basin, just as water ripples in a bounded pool. As a result, the surface ground motions of the regions close to the basin margin are amplified, which is why there are obvious edge amplification effects near the basin margin. (4) When only considering the influence of topography, there are similar PGA variation phenomena for sections I-I and III-III, that is, the PGA amplification effect is more obvious in depressions, and PGA generally decreases in a nonmonotonic manner with soil depth. Except for the above features, the ground-motion amplification effect of section III-III seems more significant, and $\mathrm{AF}_{\mathrm{PGA}}$ of section III-III is mostly larger than that of 

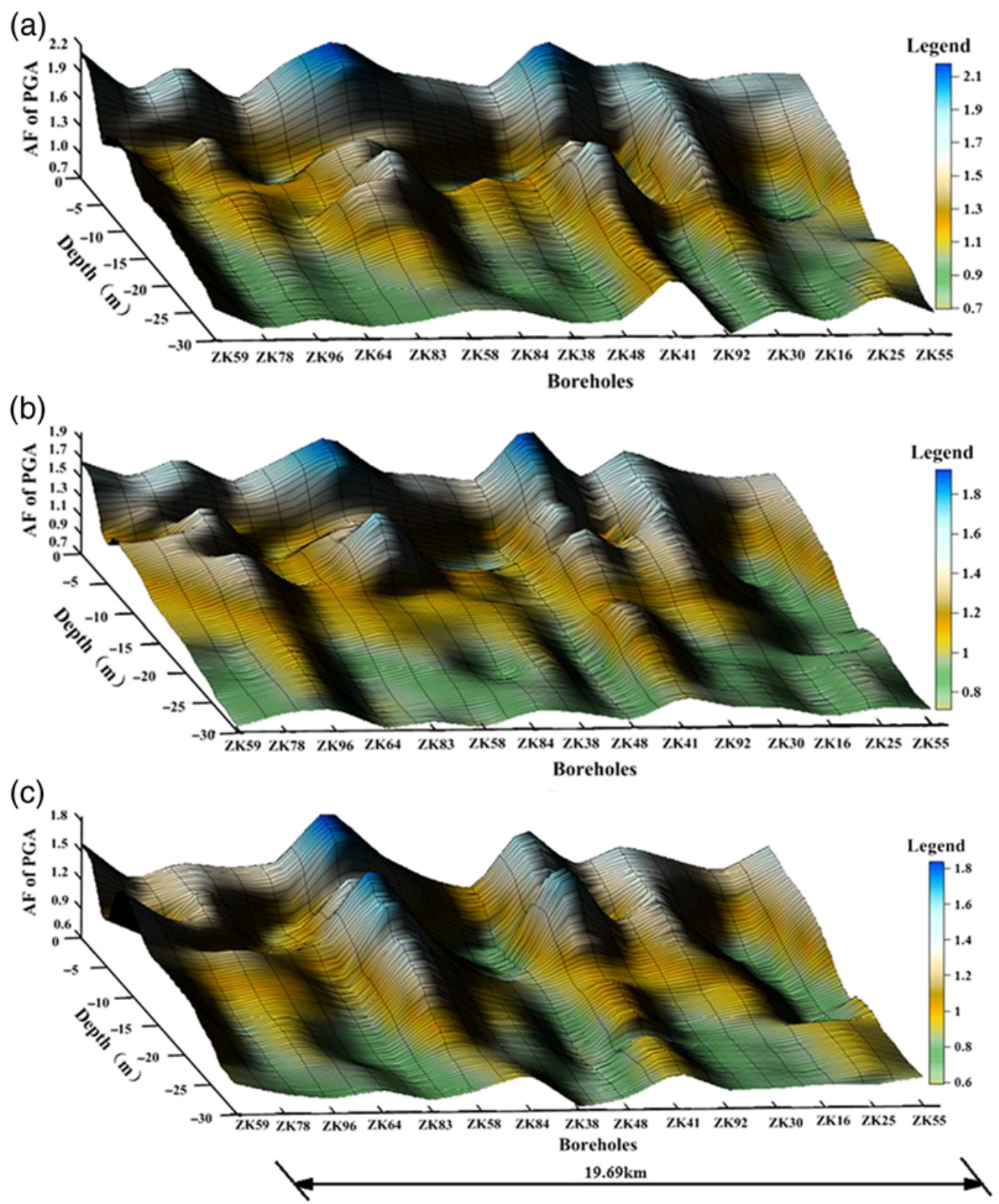

Figure 16. The PGA spatial variation of sections I-I and III-III in the Fuzhou basin for the seismic bedrock motion w1: (a) section I-I for the LLE case; (b) section I-I for the MLE case; (c) section I-I for the HLE case; (d) section III-III for the LLE case; (e) section III-III for the MLE case; and (f) section III-III for the HLE case. The color version of this figure is available only in the electronic edition.

(Continued)

section I-I. This might be influenced by the wide distribution of soft soils in section III-III, causing a soft soil amplification effect and excluding a topographic effect. Clay soils, such as those between boreholes zk46 and zk37, zk52, and zk39, are widely distributed.

Soil nonlinearity strengthened with the increased PGA of input motions observed in the simulation results. The soil behavior for LLE cases may be approximately defined as a linear condition because the maximum value of shear-strain amplitude of the soil was approximately $10^{-4}$ (actually from $10^{-6}$ to $10^{-5}$ ), whereas the maximum value of the soil shear-strain amplitudes was approximately $10^{-3}$ for cases of MLE, and
$10^{-2}$ for cases of HLE. Nonlinear soil behavior is a reasonable explanation for the reduction of PGA amplification factors.

Figure 17 shows the variation of PGA with depth for typical boreholes in section I-I for MLE cases; specific PGA variation phenomena are evident. For example, the PGA of soil layers approximately $5 \mathrm{~m}$ below the surface shows a more significant amplification effect, corresponding to the underlying bedrock interface with depression regions. However, the focusing effect on soil amplification of ground motions in the depth direction is not apparent in the soil layer of flat underlying bedrock interface regions. PGA decreases monotonically with soil depth. 

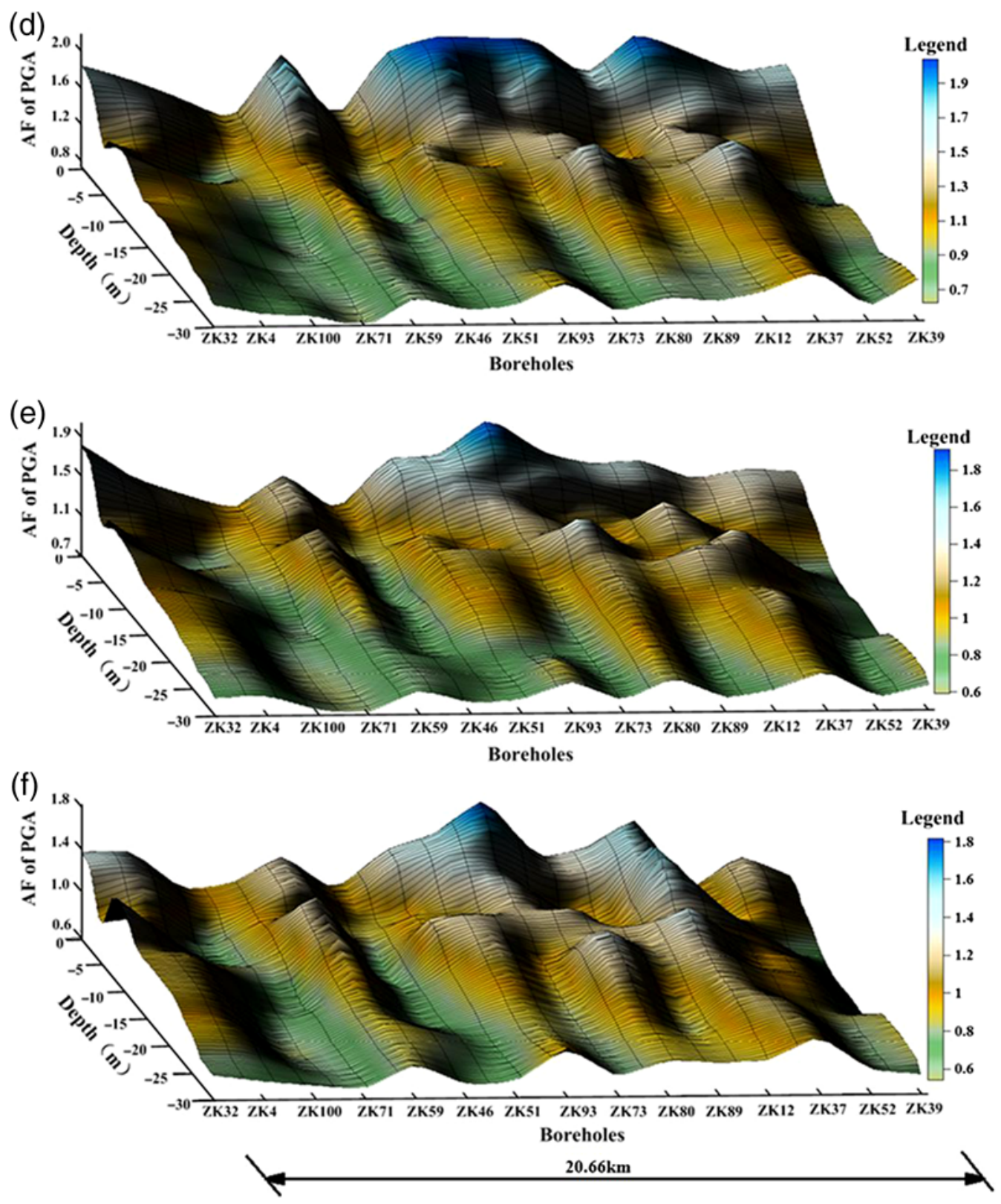

Figure 16. Continued.

\section{Spatial Variation of Spectral Accelerations}

Figure 18 shows the acceleration response spectra variation (with a damping ratio $\zeta=5 \%$ ) with soil depth at different locations in the Fuzhou basin. The acceleration response spectra of the Fuzhou basin demonstrates a two-peak or multipeak characteristic; the first peak occurs near the $0.5 \mathrm{~s}$ period, whereas the second peak is usually near the $0.8-1.5 \mathrm{~s}$ periods.

Selective amplification effects in soils near the surface may be found for input seismic bedrock motions with different frequency characteristics. Certain frequency components of input motions close to the site's predominant frequency seem to be significantly amplified, such that some frequency components of seismic waves are very abundant. There is an obvious amplification phenomenon in the periods from $0.5-1.0 \mathrm{~s}$ in the soil layer of the underlying bedrock interface flat regions (see Fig. 18a,d). However, the response spectrum shape is widened for the soil layers corresponding to the underlying bedrock interface upheaval or depression regions, and there is a movement of peak spectral acceleration in the long-period direction, thus the predominant periods of spectral shape are increased by $0.5-1.0 \mathrm{~s}$. Similarly, Figure 18 also shows the focusing effect on soil amplification on ground motion with depth.

The concept of acceleration transfer function can explain why amplification effects can be found in certain areas. The acceleration transfer function is defined as the spectral 

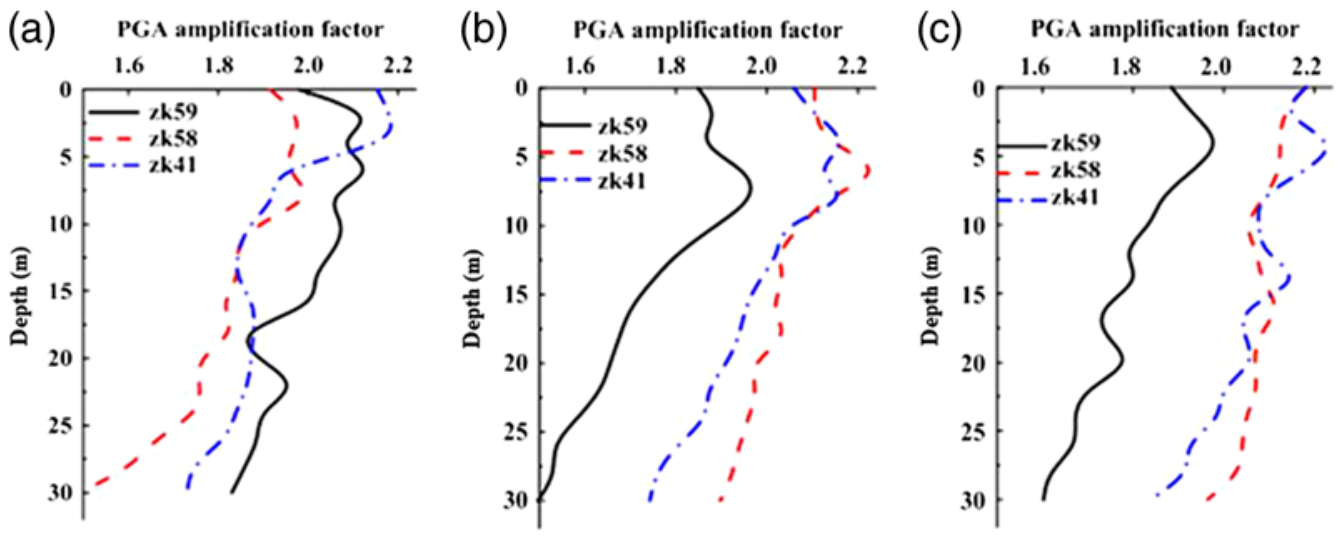

Figure 17. The PGA amplification factors with soil depth for the MLE cases: (a) w1; (b) w2; and (c) w3. The color version of this figure is available only in the electronic edition.
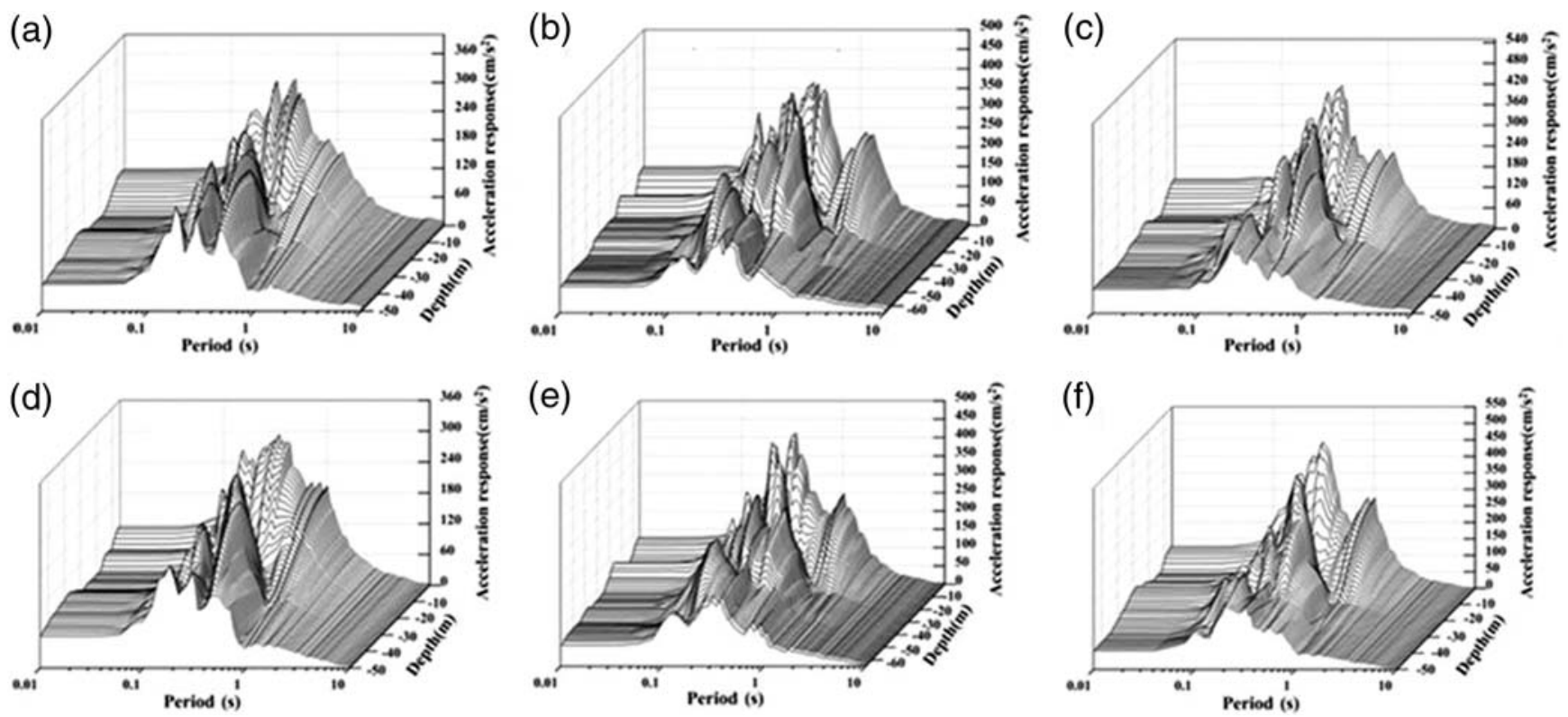

Figure 18. Spatial variation of the spectral accelerations for the MLE cases: (a) zk30 for w1; (b) zk58 for w1; (c) zk83 for w1; (d) zk30 for w2; (e) zk58 for w2; and (f) zk83 for w2.

ratio of the surface ground-acceleration Fourier spectrum to the input seismic bedrock motion-acceleration Fourier spectrum, that is, the amplification coefficient of harmonic amplitude with a different frequency during the propagation from the seismic bedrock interface to the ground surface. Figure 19 shows that the amplitude of the acceleration transfer function of the underlying bedrock interface upheaval or depression regions was relatively larger than that of underlying bedrock interface flat regions, which indicates that the corresponding site amplification effect was more obvious. Comparisons were made among Figure 19a-c, and Figure 19b,c shows a more significant low-frequency amplification effect on the soil. However, due to the serious scattering phenomenon during the seismic-wave propagation under complex geological site conditions, there is no obvious high-frequency amplification effect of the seismic bedrock motions. On the whole, in a characteristic frequency range from $0.5-2.0 \mathrm{~Hz}$, the amplification and focusing effects on soil amplification of seismic bedrock motions are relatively more significant. This may be the sensitive frequency band for the Fuzhou basin; however, when the frequency of seismic bedrock motions is smaller than $0.2 \mathrm{~Hz}$ or larger than $2.5 \mathrm{~Hz}$, the site amplification effect is not obvious.

Figure 19 shows that the values of the acceleration transfer functions for the Kobe wave seem larger than those for the Fuzhou artificial waves; additionally, simulation results indicated that the acceleration transfer functions for the six Fuzhou artificial waves were similar to each other. The difference in acceleration transfer function values between the Kobe wave and the Fuzhou artificial waves also seems more obvious for higher frequency components. Because the LLE cases may be approximately a linear elastic condition, there 
(a)

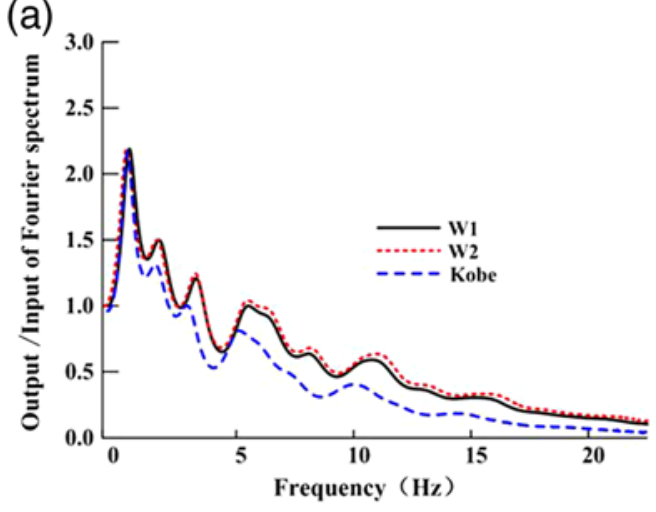

(c)

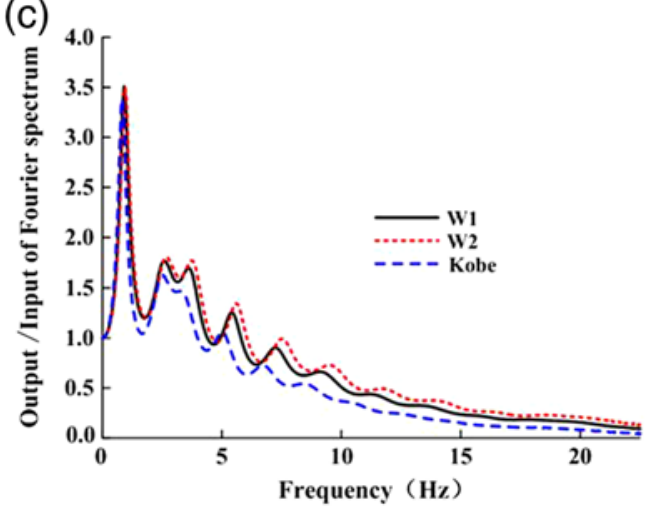

(e)

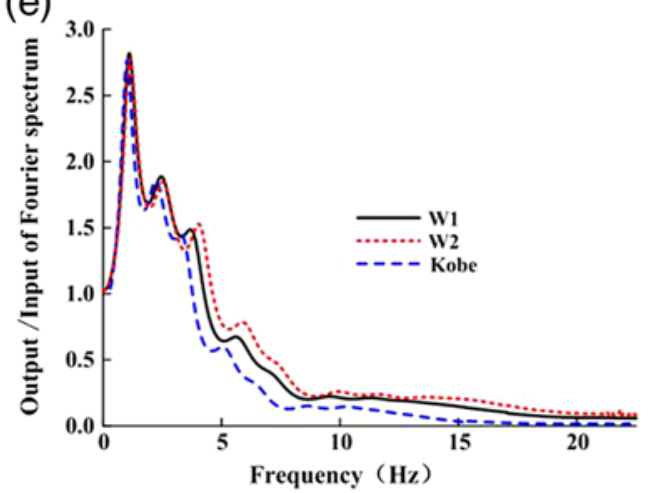

(b)

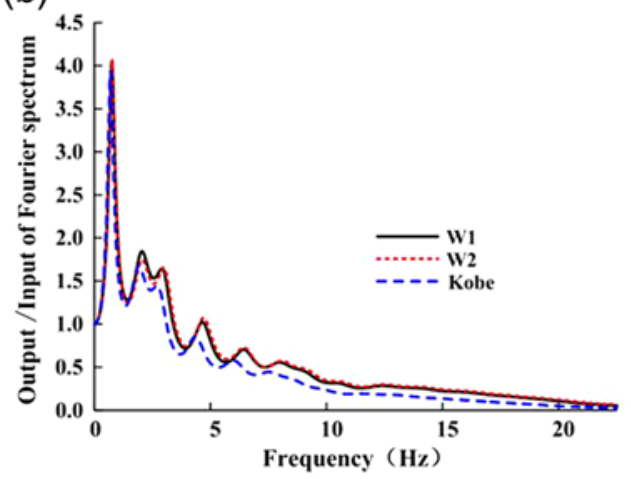

(d)

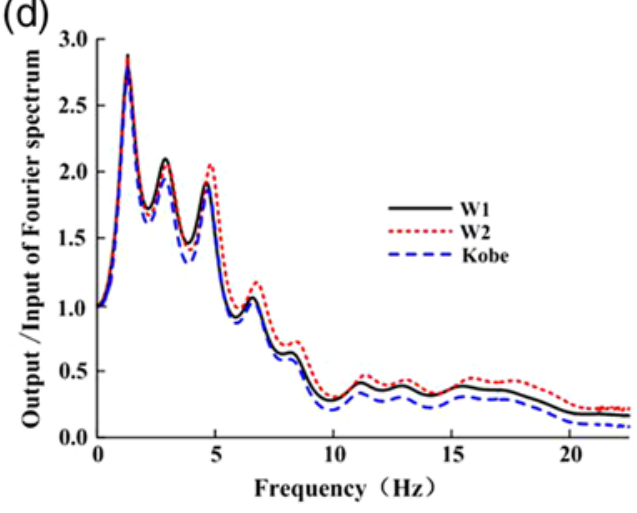

(f)

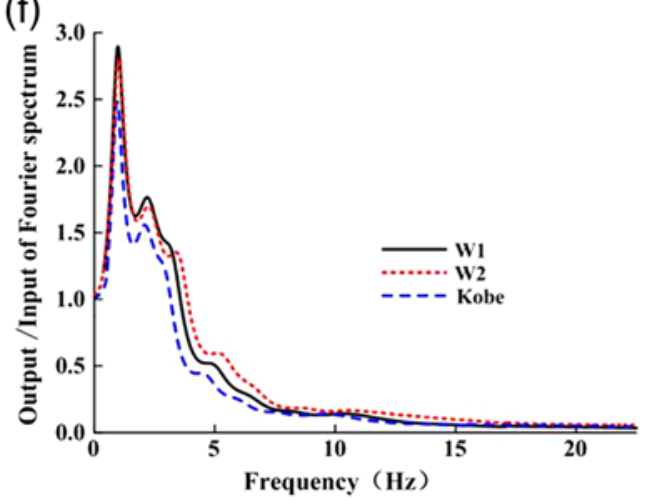

Figure 19. Surface ground-motion acceleration transfer functions: (a) zk30 for the MLE case; (b) zk58 for the MLE case; (c) zk83 for the MLE case; (d) zk16 for the LLE case; (e) zk16 for the MLE case; and (f) zk16 for the HLE case. The color version of this figure is available only in the electronic edition.

is almost no change in the acceleration transfer function because they are intrinsic to the 2D system simulation being modeled. Compared with the results for LLE cases, the nonlinear behavior of soil for the MLE cases and HLE cases is strengthened, and there are some differences for different seismic bedrock motions.

\section{Duration of Ground Motion}

One of the shortcomings of spectral acceleration is it does not reflect the influence of the duration of ground motion on engineering structures. For example, a seismic wave with a small PGA but long ground-motion duration may produce greater damage than a seismic-wave pulse with a large
PGA and short ground-motion duration. Thus, the duration is an important parameter to characterize ground motion. However, there is currently no uniform definition of the groundmotion duration. Three standards are usually adopted. The first is based on the absolute acceleration value, the second is according to the relative value of acceleration, and the third uses the accumulation of ground-motion energy. The third is adopted in this article and is based on the concept of Arias intensity (Arias, 1970). The ground-motion duration discussed in this article is considered as the duration between the 5\% and 95\% Arias intensity (Trifunac and Brady, 1975).

Figures 20-22 show the surface ground-motion duration at different borehole locations for different seismic bedrock 
(a)

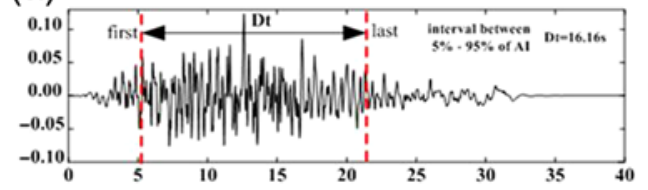

(d)

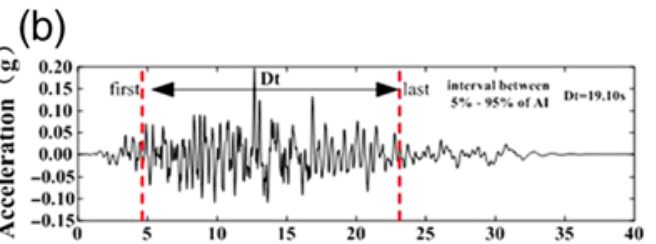

(c)
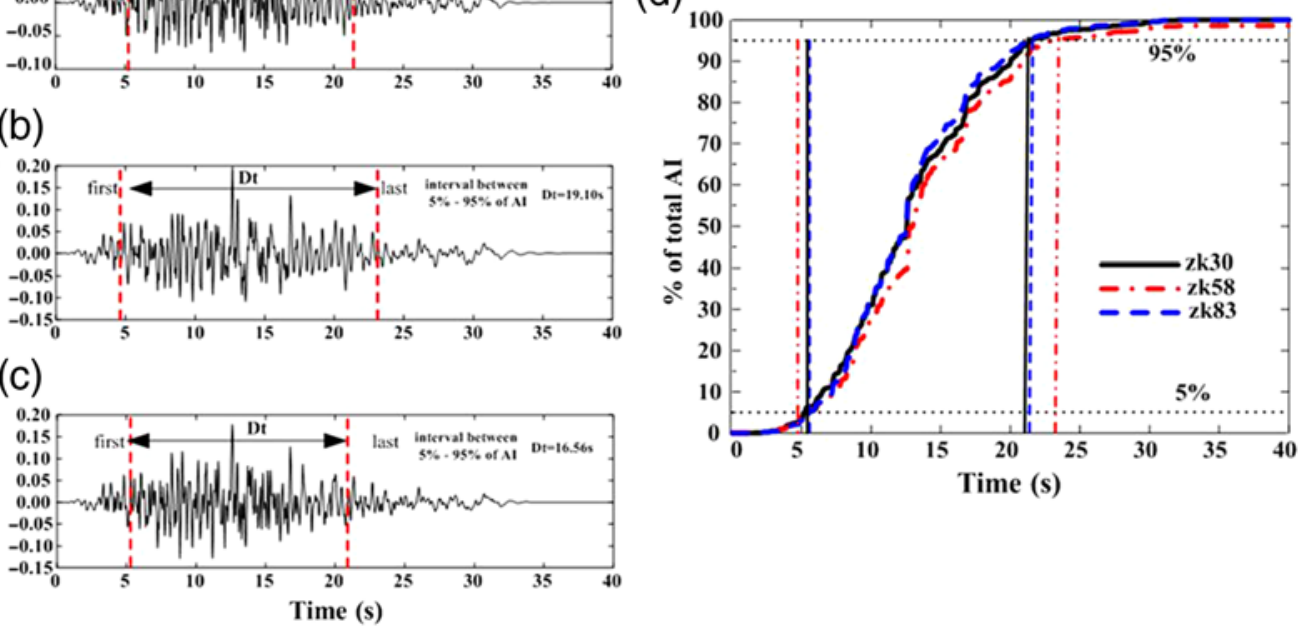

Figure 20. Surface ground-motion acceleration durations at different boreholes under input motion w1 for the MLE case: (a) zk30; (b) zk58; (c) zk83; and (d) Arias intensity (\%). The color version of this figure is available only in the electronic edition.

(a)

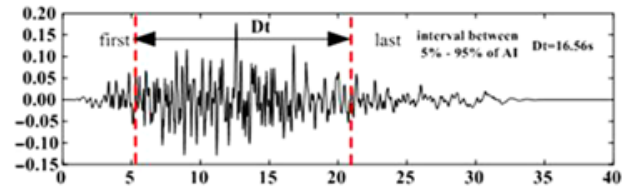

(b)

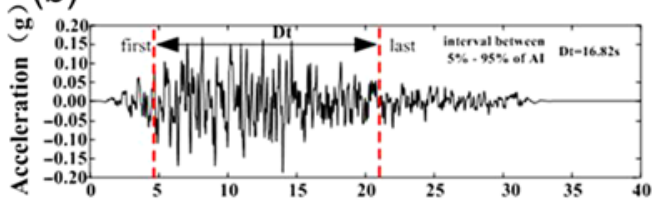

(c)

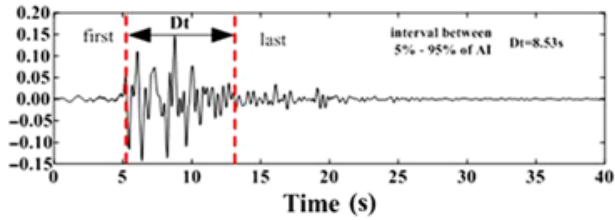

(d)

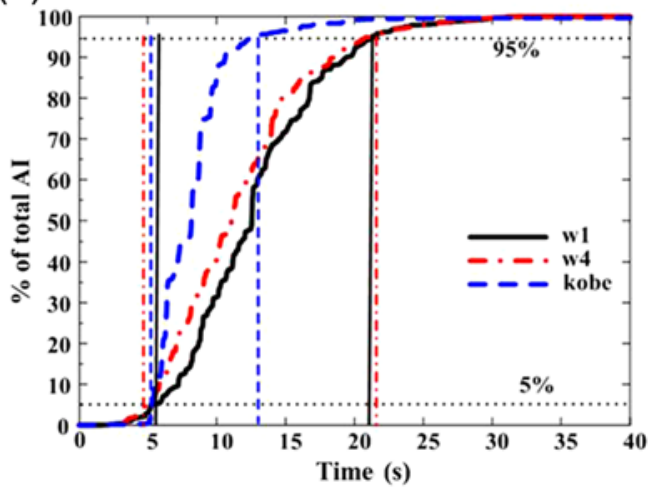

Figure 21. Surface ground-motion acceleration durations at borehole zk30 under different seismic bedrock motions: (a) w1; (b) w4; (c) Kobe wave; and (d) Arias intensity (\%). The color version of this figure is available only in the electronic edition.

motions corresponding to different level earthquakes. Figure 23 shows the durations of the seismic bedrock motions for MLE, using artificial waves w1, w2, and the Kobe wave as examples. Compared with the durations of seismic bedrock motions in a range of 14.9-15.8 s, the durations of surface ground motion are obviously prolonged, and the prolonging effect seems more pronounced with an increase in the seismic bedrock motion level. In addition, the ground surface locations may also influence the surface ground-motion duration, and the prolongation of the duration in certain locations is more apparent, such as for borehole zk58. The surface ground-motion duration at borehole $\mathrm{zk} 58$ was significantly prolonged, corresponding to the underlying bedrock interface depression region, and it was 1.46 times larger than that of the seismic bedrock motion. This was probably because the surface wave was transmitted back and forth and the seismic waves were focused in particular locations, such as the underlying bedrock interface depression regions, prolonging the dissipation time of the seismicwave propagating energy and, to some extent, prolonging the surface ground-motion duration.

The durations of the surface ground motion is dependent on the characteristics of the seismic bedrock motion (see Fig. 21), which is responsible for the surface ground-motion 
(a)

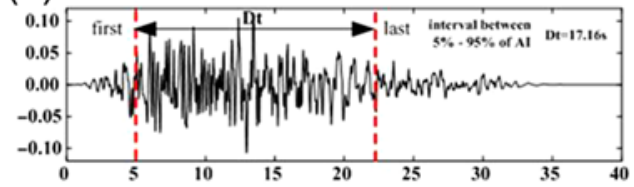

(b)

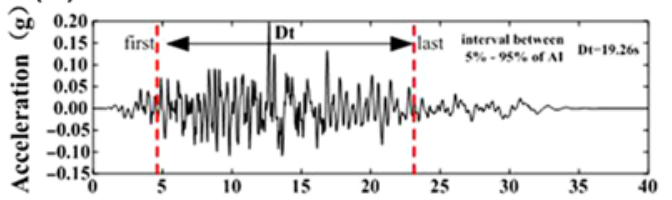

(c)

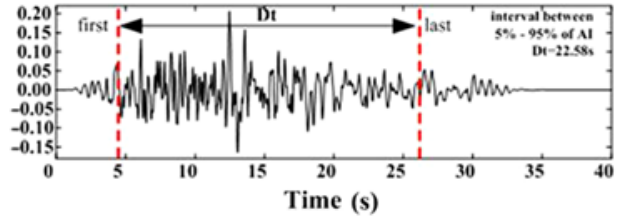

(d)

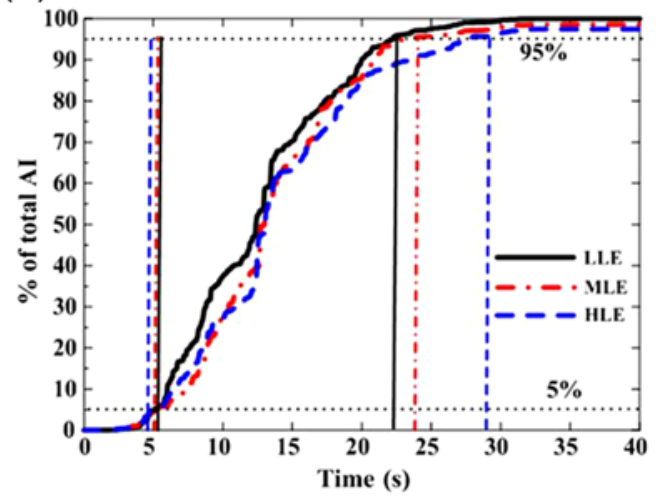

Figure 22. Surface ground-motion acceleration durations at borehole zk58 for the seismic bedrock motion w2 for (a) the LLE case; (b) the MLE case; (c) the HLE case; and (d) Arias intensity (\%). The color version of this figure is available only in the electronic edition.

(a)

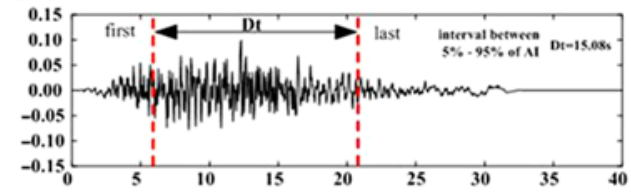

(b)

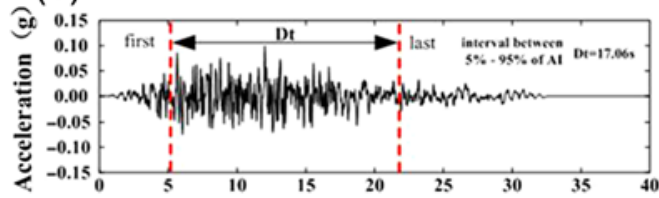

(c)

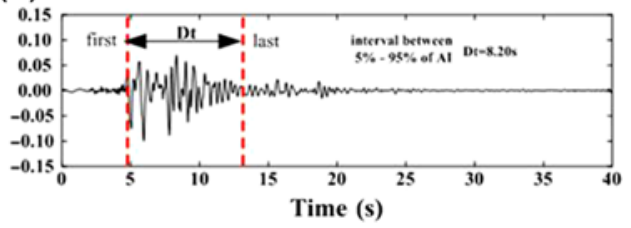

(d)

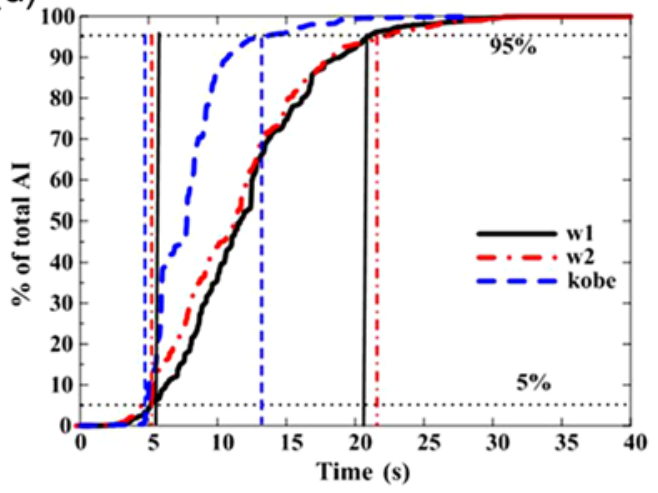

Figure 23. The input seismic bedrock motion durations for the MLE cases: (a) w1; (b) w2; (c) Kobe wave; and (d) Arias intensity (\%). The color version of this figure is available only in the electronic edition.

durations at borehole zk30 for the artificial waves w1, w4, and Kobe wave for the MLE. The surface ground-motion durations for different seismic bedrock motions vary, and the surface ground-motion durations for the artificial waves were obviously larger than that for the Kobe wave. Figure 2 shows the surface ground-motion durations at the underlying bedrock depression regions in sections I-I and III-III (such as borehole zk41 and zk93 for artificial wave w2 for the MLE) are significantly prolonged; moreover, locations close to the basin edge also have a relatively larger surface groundmotion duration. In addition, the surface ground-motion duration increases with an increase in seismic bedrock motion level (see Fig. 22). Generally, the difference of surface ground-motion duration at different locations is small for artificial wave $w 1$, whereas the difference appears larger for artificial wave w2.

\section{Contour Maps of the PGA and Predominant Period}

The PGA contour map may directly reflect the PGA spatial variation of the basin surface, and it has been used as a key basis for the precautionary urban and rural earthquake 
requirements in the Fuzhou basin. The PGA contour map was made mainly from data obtained by the 2D nonlinear analysis for the four sections in Figure 1. Furthermore, to improve the sample data coverage region, 30 boreholes in the middle region surrounded by the four sections were used for a supplementary analyses of the 1D model with Proshake. The 30 borehole locations are located at relatively flat regions of the underlying bedrock interface and have horizontal soil layers, so the results are acceptable if a 1D equivalent linear analysis is used. Figure 24a,c,e shows the average PGA contour maps of the Fuzhou basin for different level earthquakes. The average PGA maximum values of surface ground motions for LLE cases are in a range of $110-125 \mathrm{~cm} / \mathrm{s}^{2}$, corresponding to PGA amplification factors ranging from 1.4 to 2.5; the average PGA maximum values for MLE cases are $180-200 \mathrm{~cm} / \mathrm{s}^{2}$, corresponding to PGA amplification factors ranging from 1.3 to 2.0 ; and the average PGA maximum values for HLE cases are $250-300 \mathrm{~cm} / \mathrm{s}^{2}$, corresponding to PGA amplification factors ranging from 1.1 to 2.0. Generally, the average PGA amplification factor decreases with an increase in the seismic bedrock motion levels. The average PGA contour map of surface motions shows that the site amplification effects in the Fuzhou basin are generally obvious.

As there are multiple reflections of seismic waves during the propagation process, the intensity of the ground motion in certain frequency components is increased. Figure 24b,d,f shows the average predominant period contour maps for the Fuzhou basin for different seismic bedrock motions. The average predominant periods for the Fuzhou basin were $0.35-0.65 \mathrm{~s}, 0.40-0.75 \mathrm{~s}$, and $0.50-1.05 \mathrm{~s}$ for the LLE, MLE, and HLE cases, respectively. Obviously, with an increase in input motion levels, the predominant period became larger due to the stronger nonlinear behavior of the soil. This seems larger than the predominant period of the general flat sites that also belongs to site classes II and III. Generally the fluctuant of the underlying bedrock interface is in a bowl shape, and there are obvious heterogeneous soil layers; thus, the seismic site effect in the Fuzhou basin has significant nonlinear characteristics due to the focusing effect of seismicwave propagation and soil amplification, which may prolong the predominant period to some extent.

The Fuzhou basin is a typical estuarine basin with many types of sediments, and the overlying soil thickness obviously varies in different regions. Figure 25 shows the contour map of the overlying soil thickness. Taken together with Figure 24, the $\mathrm{AF}_{\mathrm{PGA}}$ of site class III is more sensitive than that of site class II to the overlying soil thickness; however, for site classes III or II, there is a similar variation tendency between the overlying soil thickness and $\mathrm{AF}_{\mathrm{PGA}}$ for the same seismic bedrock motions. The $\mathrm{AF}_{\mathrm{PGA}}$ increases with an increase in overlying soil thickness, but this correlation seems closely related to the seismic bedrock motion level. A stronger correlation occurred for seismic bedrock motion with a lower PGA, and the $\mathrm{AF}_{\mathrm{PGA}}$ of site class III seems obviously larger than that of site class II. Moreover, the influ- ence of the overlying soil thickness on the predominant period was not obvious; the prolongation of the predominant period was mainly due to the stronger nonlinear characteristics of the overlying soil.

\section{The Spectral Acceleration Amplification Factors of} Surface Motions

The spectral acceleration amplification factor of surface motion (abbreviated as $\mathrm{AF}_{\mathrm{ARS}}$ ) is defined as the spectral ratio of the acceleration response spectrum of surface motion to the acceleration response spectrum of the seismic bedrock motion. According to the $\mathrm{AF}_{\mathrm{ARS}}$ values, the spectral acceleration variation of surface motion for the Fuzhou basin can be generally observed.

Figure 26 shows the average $\mathrm{AF}_{\mathrm{ARS}}$ curves of surface motion fitted by equation (9), which was done by the least-squares method.

$$
\mathrm{AF}_{\mathrm{ARS}}=\left(2-\left(e^{-A T}-T^{2} /\left(1+T^{2}\right)\right)^{2}\right)^{B},
$$

in which $T$ is the period and $A$ and $B$ are fitting parameters.

The curve fitted between $\mathrm{AF}_{\mathrm{ARS}}$ and period $T$ has a similar shape to a smoothed spectral acceleration curve; first, with an increase in the period, the $\mathrm{AF}_{\mathrm{ARS}}$ value increases and then decreases. The peak values of the average $\mathrm{AF}_{\mathrm{ARS}}$ curve for site classes III and II were 2.5-3.0 and 2.0-2.5, respectively. The periods corresponding to the peak value of the average $\mathrm{AF}_{\mathrm{ARS}}$ curves (abbreviated as peak period) were different for site classes III and II; the peak periods for site classes III and II were $0.9-1.0 \mathrm{~s}$ and $0.6-0.8 \mathrm{~s}$, respectively. With a continuous increase in the period, the average $\mathrm{AF}_{\mathrm{ARS}}$ values gradually decrease and approximate 1.0.

\section{Discussion and Conclusions}

Comparing the results of the large-scale 2D finiteelement refined nonlinear site response analysis and the results of the 1D equivalent linear site response analyses for the Fuzhou basin, some principal differences and conclusions are as follows.

1. On the whole, the site amplification effects on the seismic bedrock motions of the $2 \mathrm{D}$ results were more obvious than those for the $1 \mathrm{D}$ results.

2. Different degrees of site amplification effects exist at different regions of the Fuzhou basin. The 2D results showed a focusing effect of seismic-wave propagation and soil amplification, both laterally and with depth, and the PGA nonmonotonically decreased with depth and had a greater focusing effect for some shallow soil layers, whereas the underlying bedrock interface significantly fluctuated (e.g., in depression regions), and edge effects on soil amplification were noted at both basin sides. Although the 1D results show that the PGA monotonically decreased with depth, it abruptly increased at a few specific depths. 

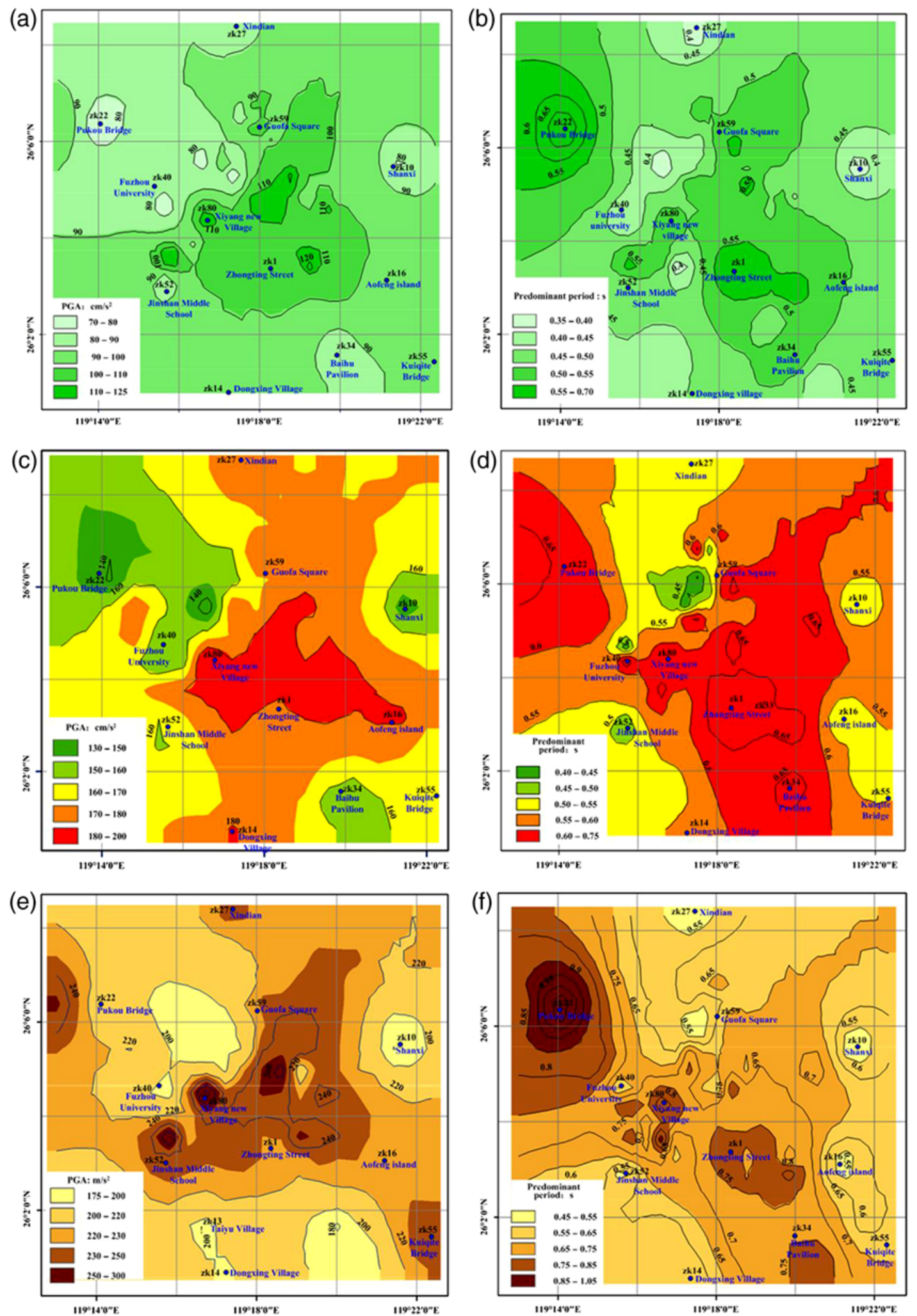

Figure 24. Average contour maps of PGA and the predominant period for the Fuzhou basin for different level earthquakes: (a) PGA for the LLE cases; (b) predominant period for the LLE cases; (c) PGA for the MLE cases; (d) predominant period for the MLE cases; (e) PGA for the HLE cases; and (f) predominant period for the HLE cases. The color version of this figure is available only in the electronic edition. 


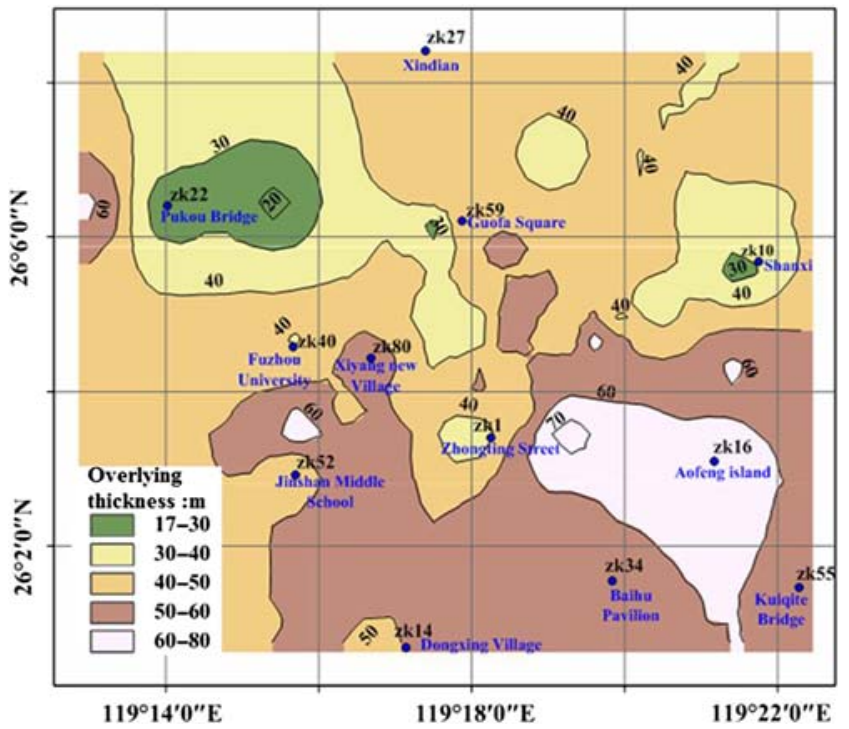

Figure 25. Overlying soil thickness contour map for the Fuzhou basin. The color version of this figure is available only in the electronic edition.

3. The predominant period of the Fuzhou basin obtained by the 2D nonlinear analysis was larger than that obtained with the equivalent 1D linear analysis. According to the 2D results, medium- and long-period site amplification effects in the Fuzhou basin were remarkable; the predominant periods of the Fuzhou basin were 0.35-0.65 s, $0.40-0.75 \mathrm{~s}$, and $0.50-1.05 \mathrm{~s}$ for low-level earthquakes, moderate-level earthquakes, and high-level earthquakes, respectively. The site amplification and focusing effects of seismic-wave propagation were more obvious in a $0.5-2 \mathrm{~Hz}$ frequency band, which is the sensitive frequency band of the seismic site response for the Fuzhou basin.

4. Surface ground-motion duration in the different locations of the Fuzhou basin were prolonged to different degrees, and the duration of the prolongation was closely related to the seismic bedrock motion characteristics. For the same seismic bedrock motion, the surface ground-motion duration increased with an increase in the input motion level. The duration prolonging effect for the $2 \mathrm{D}$ results was pronounced, which was probably due to the focusing of the propagating seismic-wave energy, and the constant reflection and refraction of the seismic-wave might prolong the surface ground duration.

5. Average contour maps for the PGA and predominant period of the Fuzhou basin were drawn, and spectral acceleration amplification factors of the surface motion were also recommended. The ground-motion parameters proposed in this article could be used as a key basis for the precautionary urban and rural earthquake requirements for the Fuzhou basin.

To some degree, the large-scale 2D finite-element refined nonlinear analysis could truly reflect the site amplification effects for some frequency components of the ground motion. It also shows a better simulating ability of the focusing effect of seismic-wave propagation by soil amplification. (a)

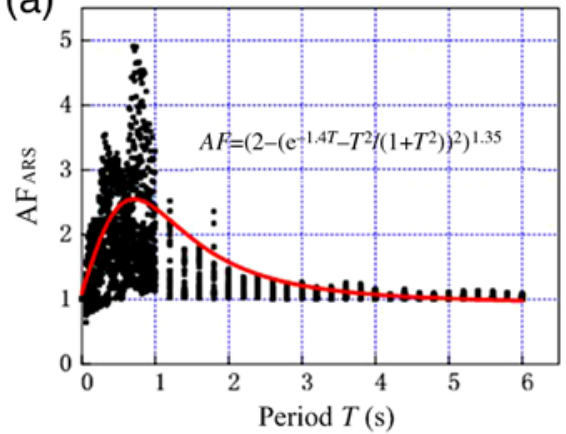

(d)

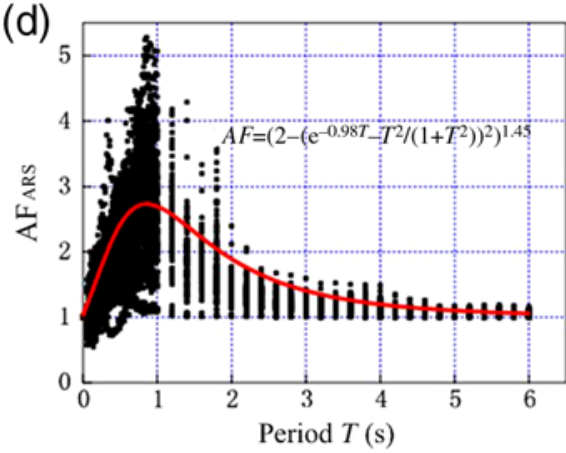

(b)

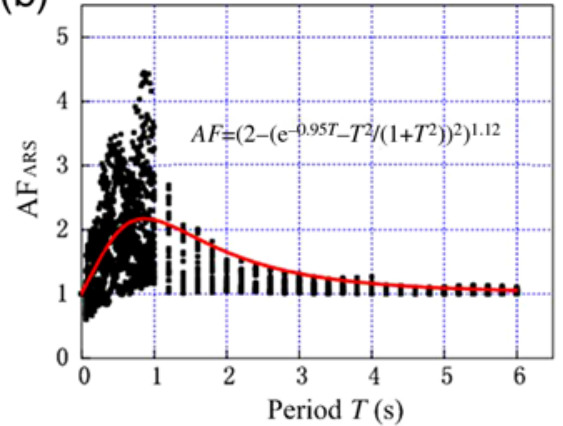

(e)

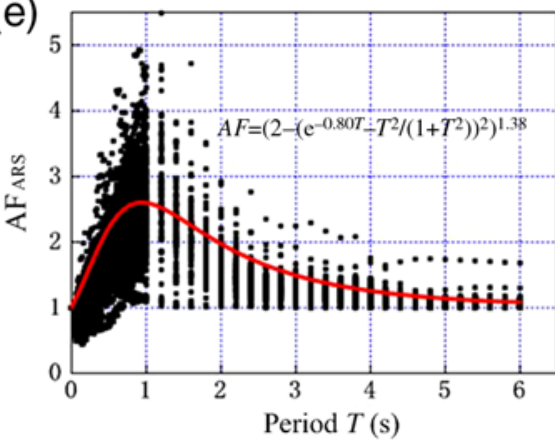

(c)

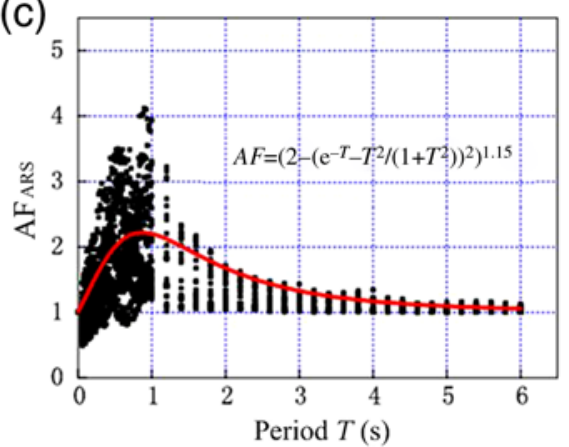

(f)

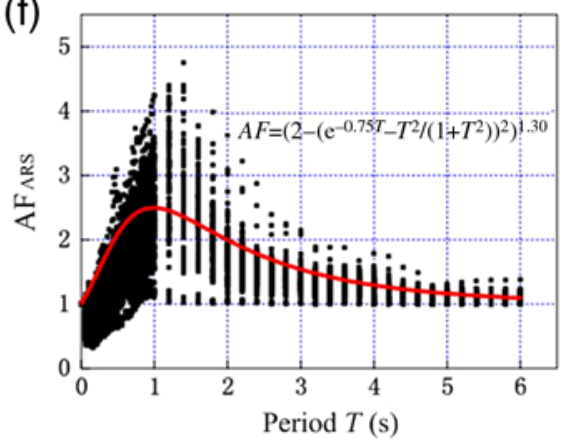

Figure 26. Amplification factor fitting curves of surface ground-motion spectral accelerations (5\% damping ratio): (a) site class II for the LLE case; (b) site class II for the MLE case; (c) site class II for the HLE case; (d) site class III for the LLE case; (e) site class III for the MLE case; (f) site class III for the HLE case. The color version of this figure is available only in the electronic edition. 
In general, use of the 1D equivalent linear wave propagation models may be unadvisable when the lateral soil spatial variation is not homogeneous and the underlying bedrock interface is obviously variable.

All of the conclusions above are subject to the given calculation assumptions. Ground-motion spatial variability in a basin is influenced by many factors, such as microtopography, soil lateral heterogeneity, and nonlinear soil behavior caused by seismic bedrock motions. The focusing effect of seismic-wave propagation in a basin with a significant fluctuant of underlying bedrock interface warrants more detailed research. Seismic effects in the basin should be fully considered in urban earthquake disaster reduction planning and in the seismic design of engineering projects.

\section{Data and Resources}

The artificial earthquake waves, the borehole data of the Fuzhou basin (including latitude and longitude data), the shear-wave velocity, and the disturbed soil samples for dynamic test were provided by the Seismological Bureau of Fujian Province, China. The normalized shear modulus and damping ratio curves of typical soil samples of the Fuzhou basin were tested by the authors with a free vibration column apparatus, and the rest of the data used in this article, such as the introduction of the seismic environment, came from the published sources listed in the references.

\section{Acknowledgments}

We thank National Program on Key Basic Research Projects of China (2011CB013601) and the National Natural Science Foundation of China (51378258) for support. This research is also based on reliable and detailed information provided by the Seismological Bureau of Fujian Province, and we are grateful for this support; the help of Huiteng Cai and Jinfu Zhang of the Seismological Bureau of Fujian Province is greatly appreciated. Thanks also go to the reviewers and editors of this article who helped us improve the work.

\section{References}

Arias, A. (1970). A measure of earthquake intensity, in Seismic Design for Nuclear Power Plants, R. J. Hansen (Editor), MIT Press, Cambridge, Massachusetts, 438-483.

Assimaki, D., and S. Jeong (2013). Ground-motion observations at Hotel Montana during the M 7.0, 2010 Haiti Earthquake: Topography or soil amplification, Bull. Seismol. Soc. Am. 103, no. 5, 2577-2590.

Assimaki, D., C. Ledezma, G. A. Montalva, and A. Tassara (2012). Site effects and damage patterns, special issue on the Maule 8.8 earthquake, Earthq. Spectra 28, no. 1, 55-74.

Bard, P. Y., M. Campilo, F. J. Chavez-Garcia, and F. J. Sanchez-Sesma (1988). The Mexico earthquake of September 19, 1985-A theoretical investigation of large- and small-scale amplification effects in the Mexico City valley, Earthq. Spectra. 4, 609-633.

Campillo, M., J. C. Gariel, K. Aki, and F. J. Sánchez-Sesma (1989). Destructive strong ground motion in Mexico city: Source, path, and site effects during great 1985 Michoacán earthquake, Bull. Seismol. Soc. Am. 79, no. $6,1718-1735$.

Chávez-García, F. J., D. Raptakis, K. Makrab, and K. Pitilakis (2000). Site effects at Euroseistest-II. Results from 2D numerical modeling and comparison with observations, Soil Dynam. Earthq. Eng. 19, 23-39.
Chen, G., and H. Zhuang (2005). Developed nonlinear dynamic constitutive relations of soils based on Davidenkov skeleton curve, Chin. J. Geotech. Eng. 27, no. 8, 860-864 (in Chinese).

Chen, G., L. Chen, L. Jing, and H. Long (2011). Comparison of implicit and explicit finite element methods with parallel computing for seismic response analysis of metro underground structures, J. China Railway Soc. 33, no. 11, 112-117 (in Chinese).

Chinese Standard (2005). Evaluation of Seismic Safety for Engineering Sites issued by General Administration of Quality Supervision, Inspection and Quarantine of the People's Republic of China, Beijing, GB177412005, 57-74 (in Chinese).

Chinese Standard (2010). Code for Seismic Design of Buildings issued by Ministry of Housing and Urban-Rural Development of the People's Republic of China, Beijing, GB50011-2010, 19-20 (in Chinese).

Di Fiore, V. (2010). Seismic site amplification induced by topographic irregularity: Results of a numerical analysis on 2D synthetic models, Rev. Eng. Geol. 114, nos. 3/4, 109-115.

Gélis, C., and L. F. Bonilla (2012). 2D-P-SV numerical study of soilsource interaction in a non-linear basin, Geophys. J. Int. 191, no. $3,1374-1390$.

Gélis, C., and L. F. Bonilla (2014). Influence of a sedimentary basin infilling description of the 2D P-SV wave propagation using linear and nonlinear constitutive models, Geophys. J. Int. 198, no. 3, 1684-1700.

Héloïse, C. (2012). Site effect assessment using KiK-net data: Part 1. A simple correction procedure for surface/downhole spectral ratios, Bull. Earthq. Eng. 10, 421-448.

Hu, Y., and X. He (1986). Phase angle consideration in generating response spectrum-compatible ground motion, Earthq. Eng. Eng. Vib. 6, no. 2, 37-51 (in Chinese).

Lanzo, G., and A. Pagliaroli (2012). Seismic site effects at near-fault strongmotion stations along the Aterno River Valley during the $M_{\mathrm{w}} 6.32009$ L'Aquila earthquake, Soil Dynam. Earthq. Eng. 40, 1-14.

Lanzo, G., F. Silvestri, A. Costanzo, A. D'Onofrio, L. Martelli, A. Pagliaroli, S. Sica, and A. Simonelli (2011). Site response studies and seismic microzoning in the Middle Aterno valley (L'Aquila, Central Italy), Bull. Earthq. Eng. 9, 1417-1442.

Liu, J., and B. Li (2005). A unified viscous-spring artificial boundary for 3-D static and dynamic applications, Sci. China Earth Sci. 35, no. 9, 966980 (in Chinese).

Liu, J., Y. Gu, and Y. Du (2006). Consistent viscous-spring artificial boundaries and viscous-spring boundary elements, Chin. J. Geotech. Eng. 28, no. 9, 1070-1075 (in Chinese).

Makraa, K., F. J. Chávez-García, D. Raptakis, and K. Pitilakis (2005). Parametric analysis of the seismic response of a $2 \mathrm{D}$ sedimentary valley: Implications for code implementations of complex site effects, Soil Dynam. Earthq. Eng. 25, no. 4, 303-315.

Martin, P. P., and H. B. Seed (1982). One-dimensional dynamic ground response analysis, J. Geotech. Eng. 7, 935-952.

Moczo, P. (1989). Finite-difference technique for $S H$ waves in 2D media using irregular grids: Application to the seismic response problem, Geophys J. Int. 99, 321-329.

Moczo, P., and P.-Y. Bard (1993). Wave diffraction, amplification and differential motion near strong lateral discontinuities, Bull. Seismol. Soc. Am. 83, 85-106.

Moczo, P., P. Labák, J. Kristek, and F. Hron (1996). Amplification and differential motion due to an antiplane $2 \mathrm{D}$ resonance in the sediment valleys embedded in a layer over the half-space, Bull. Seismol. Soc. Am. 86, 1434-1446.

Qian, P., Y. Shen, D. Guo, and X. Xie (1984). Parts of seismic hazard in the Tonghai earthquake and their analysis, J. Seismol. Res. 7, no. 3, 357-363 (in Chinese).

Rubinstein, J. L. (2011). Nonlinear site response in medium magnitude earthquakes near Parkfield, California, Bull. Seismol. Soc. Am. 101, no. $1,275-286$

Seed, H. B., M. P. Romo, and J. I. Sun (1988). Relationships between soil conditions and earthquake ground motions, Earthq. Spectra. 4, 687-729. 
Semblat, J. F., P. Dangla, M. Kham, and A. M. Duval (2002). Seismic site effects for shallow and deep alluvial basins: In-depth motion and focusing effect, Soil Dynam. Earthq. Eng. 22, 849-854.

Semblat, J. F., A.-M. Duval, and P. Dangla (2000). Numerical analysis of seismic wave amplification in Nice (France) and comparisons with experiments, Soil Dynam. Earthq. Eng. 19, 347-362.

Sheng, Z., Y. Lu, and Y. Shi (2013). Seismic response analysis of valley topography, China Earthq. Eng. J. 35, no. 1, 126-132 (in Chinese).

Singh, S. K., E. Mena, and R. Castro (1988). Some aspects of source characteristics of the 19 September 1985 Michoacan earthquake and ground motion amplification in and near Mexico City from strong motion data, Bull. Seismol. Soc. Am. 78, no. 2, 451-477.

Somerville, P. G., and R. Graves (1993). Conditions that give rise to unusually large long period ground motions, The Structural Design of Tall Buildings 2, no. 3, 211-232.

Stewart, J. P., S. J. Chiou, J. D. Bray, R. W. Graves, P. G. Sommerville, and N. A. Abrahamson (2001). Ground motion evaluation procedures for performance-based design, PEER Report 9, 57-85.

Sun, C.-G., and C.-K. Chung (2008). Assessment of site effects of a shallow and wide basin using geotechnical information-based spatial characterization, Soil Dynam. Earthq. Eng. 28, 1028-1044.

Trifunac, M. D., and A. G. Brady (1975). A study on the duration of strong earthquake ground motion, Bull. Seismol. Soc. Am. 65, no. 3, 581-626.

Wen, X., and X. Xu (2003). Seismogenic environment and assessment of the maximum magnitude of potential earthquakes on the main faults in Fuzhou Basin, Seismol. Geol. 25, no. 4, 510-522 (in Chinese).

Wood, H. O. (1916). California earthquakes: A synthetic study of recorded shocks, Bull. Seismol. Soc. Am. 6, nos. 2/3, 55-180.

Zhan, J. (2012). Nonlinear seismic effect of deep soft site under far-field large earthquake, Ph.D. Thesis, Nanjing Technical University, Nanjing, China (in Chinese).
Zheng, G. (2013). Analysis on geological factors of exploiting underground space resources in Fuzhou City, Chin. J. Underground Space and Eng. 9, no. 1, 13-17 (in Chinese).

Zheng, R., G. Chen, X. Xu, and J. Zhu (2005). Strata division of buried late quaternary of Fuzhou Basin, Seismol. Geol. 27, no. 4, 556-562 (in Chinese).

Institute of Geotechnical Engineering

Nanjing Tech University

Nanjing

Jiangsu 21009, China

(G.C., D.J., J.Z.)

Department of Mechanical and Civil Engineering

California Institute of Technology

Pasadena, California 91125

(J.S.)

Institute of Geophysics

China Earthquake Administration

Beijing 100081, China

(X.L.)

Manuscript received 30 March 2014;

Published Online 3 February 2015 